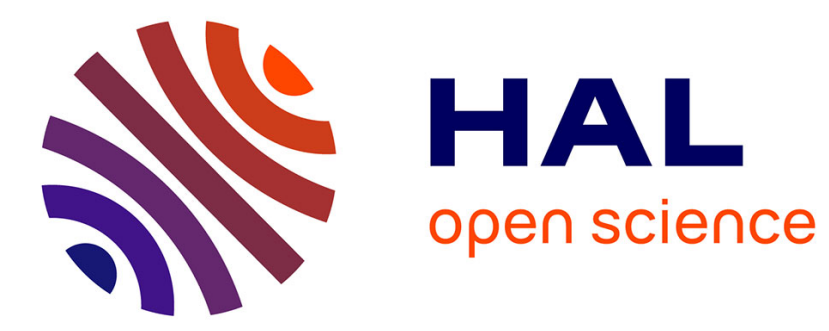

\title{
Dynamique de spin et interactions spin-photon
}

H. Le Gall

\section{- To cite this version:}

H. Le Gall. Dynamique de spin et interactions spin-photon. Revue de Physique Appliquée, 1974, 9

(5), pp.793-818. 10.1051/rphysap:0197400905079300 . jpa-00243846

\section{HAL Id: jpa-00243846 https://hal.science/jpa-00243846}

Submitted on 1 Jan 1974

HAL is a multi-disciplinary open access archive for the deposit and dissemination of scientific research documents, whether they are published or not. The documents may come from teaching and research institutions in France or abroad, or from public or private research centers.
L'archive ouverte pluridisciplinaire HAL, est destinée au dépôt et à la diffusion de documents scientifiques de niveau recherche, publiés ou non, émanant des établissements d'enseignement et de recherche français ou étrangers, des laboratoires publics ou privés. 


\title{
DYNAMIQUE DE SPIN ET INTERACTIONS SPIN-PHOTON
}

\author{
H. LE GALL \\ Laboratoire de Magnétisme et d'Optique des Solides, \\ C. N. R. S., 92190 Meudon-Bellevue, France
}

\begin{abstract}
Résumé. - L'évolution d'un système de spin para- ou ferromagnétique est revue à partir d'une description unifiée des différentes interactions spin-photon, spin-spin et spin-phonon. On discute principalement les mécanismes de base de manière simple et physique en tenant compte de la nature dipolaire électrique ou magnétique et du caractère réel ou virtuel de ces interactions. Dans la bande microonde l'évolution des spins dépend de la géométrie du pompage (perpendiculaire ou parallèle), du niveau d'excitation (évolutions linéaire, non linéaire, paramétriques) et du régime stationnaire ou transitoire. Deux cas de régime transitoire ou impulsionnel sont discutés. L'un est à la base de l'écho de spin et le second décrit l'évolution adiabatique ou non adiabatique (apparition de spinflip) d'un ensemble de spins dans un champ magnétique variable en direction. Dans un ferromagnétique, le spin-flip produit des magnons qui augmentent le temps de basculement de l'aimantation. Après description des ondes de spin et des magnons dans les ferro- et antiferromagnétiques, les interactions magnons-magnons, magnons-phonons et magnons-impuretés qui sont à la base de la relaxation magnétique seront analysées. Dans la bande optique les interactions spin-photon définissent de multiples effets $M$. O., soit du type absorption (absorption à 2 magnons et magnonphonon, absorption exciton-magnon ou « magnon side-bands »), soit du type diffusion, ou élastique (effets Faráday et Cotton-Mouton), ou inélastique (effets Raman à 1, 2 et 4 magnons). Les relations physiques et analytiques qui existent entre les différents effets magnéto optiques seront précisées en introduisant les dipôles électriques et magnétiques induits par la lumière soit dans les équations de Maxwell (description macroscopique classique), soit dans les Hamiltoniens d'interactions dipolaires électriques et magnétiques (description macroscopique en seconde quantification). Dans une dernière partie l'origine microscopique des interactions spin-photon est discutée à partir des transitions réelles et virtuelles qui apparaissent dans l'expression de la polarisabilité dynamique tensorielle d'un ion magnétique en présence des couplages spin-orbite et d'échange.
\end{abstract}

\begin{abstract}
A survey of the para- and ferromagnetic spins evolution is given from an unified description of the different spin-photon, spin-spin and spin-phonon interactions. The basis mechanisms are discussed in detail by using the real and virtual character of the electric and magnetic dipole interactions. In the microwave range the spins evolution depends on the pumping geometry (perpendicular or parallel), on the excitation level (linear, non-linear and parametric excitation), on the transient or steady-state pumping type. Two transient or pulse evolutions are discussed : one is the basis of the spin-echo technique and the second describes the adiabatic or non-adiabatic (spin-flip) evolution of a spins system in a d. c. magnetic field having a change of its direction. In a ferromagnet the spin-flip induces magnons which increase the reversal magnetization time. After the description of the spin-waves and magnons in the ferro- and antiferromagnets, the magnon-magnon, magnon-phonon and magnon-magnetic impurities interactions are analyzed. In the optical range the spin-photon interactions induce many magnetooptical (M. O.) effects, either absorption type (2-magnon and magnon-phonon absorption, magnon-side-bands), or elastic scattering type (Faraday and Cotton-Mouton effects) and inelastic scattering type (1, 2 and 4-magnon Raman effects). The physical and analytical relations between the different $M$. $O$. effects are obtained by introducing the electric and magnetic dipoles induced by the light, either in the Maxwell equations (classical macroscopic description), or in the electric and magnetic dipole interactions Hamiltonians (second-quantization macroscopic description). In the last part the microscopic origin of the spin-photon interactions is discussed from the real and virtual transitions which appear in the expression of the tensorial dynamical polarizability of a magnetic ion with the spin-orbit and exchange couplings.
\end{abstract}

1. Introduction. - Nous décrivons l'évolution d'un système de spins en interaction avec un rayonnement électromagnétique, en limitant l'étude au cas des cristaux paramagnétiques, ferro-, ferri- et antiferromagnétiques. La grande variété de phénomènes observés est associée d'une part aux différents types de transitions spin-photon, spin-spin et spin-phonon qui apparaissent dans les cristaux magnétiques et d'autre part au caractère réel ou virtuel et à la nature dipolaire électrique ou dipolaire magnétique de ces transitions. Comme indiqué sur la figure 1 les couplages réels et virtuels du rayonnement aux différents états localisés (spins, électrons et noyaux) et collectifs (phonons, magnons, "excitons, hélicons...) du cristal 


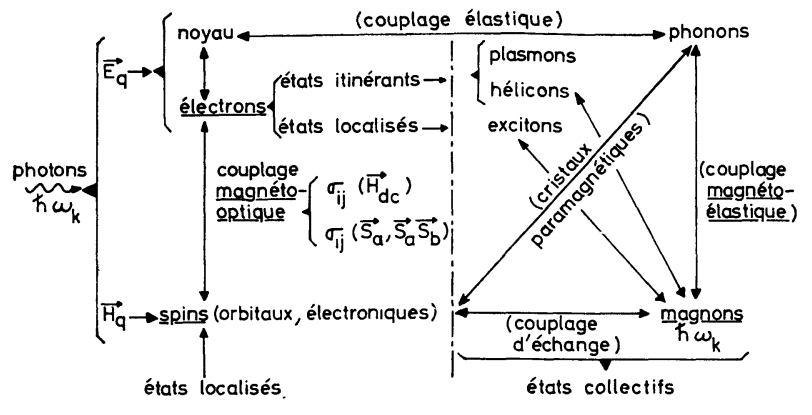

Fig. 1. - Transfert d'énergie d'un champ électromagnétique vers les différents états d'un cristal magnétique.

magnétique apparaît par le biais des interactions dipolaires magnétique et électrique et des interactions d'échange, magnétooptiques, photoélastiques et magnétoélastiques. Ces effets peuvent être séparés soit à partir des transitions dipolaires électriques et magnétiques, soit à partir des processus d'absorption et de dispersion, soit encore à partir des systèmes à 1 ion ou à 2 ions. Tous ces processus peuvent enfin être répartis entre deux sous-groupes relatifs aux interactions du premier et du second ordre en composantes de l'aimantation (description macroscopique) ou d'opérateur de spin (description microscopique).

La figure 2 montre en fonction de la longueur d'onde les bandes d'apparition de la plupart des interactions spin-photon connues jusqu'ici depuis la première observation de l'effet Faraday en 1845. Le couplage dipolaire magnétique est responsable des résonances bien connues dans les composés para-, ferro-, ferriet antiferromagnétiques (ou résonance d'échange). Nous donnerons dans le chapitre II une description classique et quantique simple de ces phénomènes qui sont basés sur l'effet gyromagnétique.

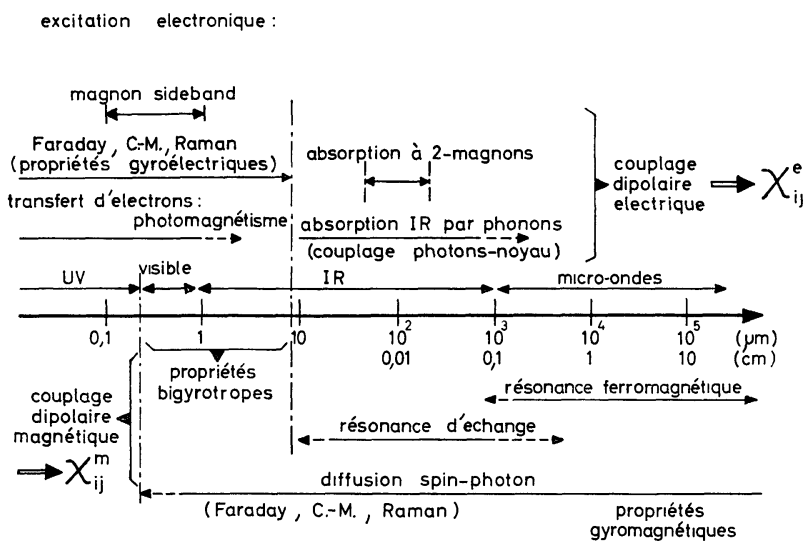

FIG. 2.

Nous verrons entre autres que si la résonance magnétique apparaît en général dans la gamme microonde, l'effet dispersif qui lui est associé peut encore induire dans la bande optique des transitions virtuelles comme les effets Faraday, Cotton-Mouton et Raman de spin. L'importance des transitions réelles et virtuelles sera précisée pour expliquer les phénomènes d'absorption et de dispersion. Nous verrons que l'évolution des spins soumis à une excitation radiofréquence est décrite à partir du couplage dipolaire magnétique et dépend de la géométrie du pompage ( " perpendiculaire » ou " parallèle »), du niveau d'excitation (évolution linéaire, non linéaire, paramétrique) et du type de pompage en régime continu ou transitoire. En régime transitoire nous analyserons deux cas intéressants. Le premier concerne une excitation radiofréquence ( $r$. f.) en régime impulsionnel à haut niveau qui est à la base du phénomène d'écho et spin si précieux pour la mesure des temps de relaxation spin-spin et spin-réseau en résonances électroniques et nucléaires. Le second est associé à l'évolution d'un ensemble de spins soumis à un champ magnétique d'amplitude constante mais pouvant brusquement changer de direction. Cette évolution est soit adiabatique, soit non adiabatique si le temps de commutation de la direction du champ statique est lente ou rapide comparée à la fréquence de Larmor des spins. Dans le cas d'une évolution non adiabatique le système de spins se trouve dans un état excité après basculement du champ directeur. Dans un système de spins couplés par échange le basculement de l'aimantation est dans ce cas accompagné par l'émission d'un "nuage " d'ondes de spin qui augmente le temps de basculement.

Après la description des modes d'oscillations magnétiques dans les ferro- et antiferromagnétiques nous préciserons les interactions magnons-magnons, magnons-phonons et magnons-impuretés magnétiques qui sont à la base de la relaxation ferro- et ferrimagnétique.

Comme indiqué sur la figure 2, les interactions spinphoton induites par le couplage dipolaire électrique apparaissent principalement dans la bande optique IR, visible et U. V. En plus des effets Faraday, CottonMouton et Raman de spin déjà signalés, de nouveaux processus ont été découverts récemment en utilisant les techniques lasers : ce sont les absorptions à 2 magnons dans l'infrarouge lointain, les bandes latérales de magnon (ou interactions exciton-magnon) et les effets photomagnétiques qui sont basés sur des transferts de charges électroniques. Ces interactions qui appartiennent à la famille des effets magnétooptiques seront discutées dans le chapitre III. En partant des dipôles électriques et magnétiques induits par la lumière, nous déduirons avec les équations de Maxwell les modes propres de propagation électromagnétiques dans un cristal magnétique. Nous préciserons les biréfringences magnétiques circulaire (B. M. C.) et linéaire (B. M. L.) correspondant aux effets M. O. des premier et second ordres. Dans une seconde étape nous déduirons les termes énergétiques classiques et quantiques qui montrent la possibilité de connecter les diffusions spin-photon élastiques (effets Faraday et Cotton-Mouton) aux diffusions inélastiques (effets Raman à 1 et 2 magnons. Dans une dernière section 
nous analyserons la description microscopique quantique des effets $M$. O.

2. Mouvement de spin dans la bande radiofréquence : gyromagnétisme. - 2.1 EFFET GYROMAGNÉTIQUE : PRÉCESSION DE LARMOR. EVOLUTION DE L'AIMANTATION DANS UN RÉFÉRENTIEL TOURNANT. Un ensemble de spins nucléaires ou électroniques évoluent par l'intermédiaire du couplage des moments magnétiques qui leur sont associés avec un champ extérieur comme les champs magnétiques, électromagnétiques (ou photons) et élastiques (ou phonons). A cet effet nous rappelons les nombres quantiques $n$, $l, m_{l}, s, m_{s}$ qui caractérisent les états d'un électron de la couche $n$. Le mouvement de cet électron défini par les moments cinétiques orbital $\mathbf{G}_{l}$ et de $\operatorname{spin} \mathbf{G}_{\boldsymbol{s}}$ de modules

$$
\sqrt{l(l+1)} \cdot \hbar \text { et } \sqrt{s(s+1)} \cdot \hbar(=\sqrt{3 / 4} \hbar)
$$

respectivement. Le couplage spin-orbite se traduit par le vecteur résultant $\mathbf{j}=\mathbf{l}+\mathbf{s}$ dont le module est défini par $\sqrt{j(j+1)} . \hbar$ avec $|l-s|<j<l+s$. Les évolutions orbitale et de spin de l'électron ayant une charge non nulle se traduisent par l'existence d'un moment magnétique électronique $\mathbf{M}$ tel que :

$$
\mathbf{M}_{l}=\gamma_{l} \mathbf{G}_{l} \quad \text { et } \quad \gamma=-g_{j}\left(\frac{e}{2 m c}\right)
$$

où $\gamma, g_{j}$ sont le facteur gyromagnétique et le facteur de Landé. La valeur de $g_{j}$ dépend des contributions relatives de $\mathbf{l}$ et $\mathbf{s}$ au moment cinétique total $\mathbf{j}$, c'est-àdire qu'il dépend de la nature du couplage spin-orbite. $g_{j}$ est égal à 1 et 2 dans les cas limites où $s=0$ et $l=0$ respectivement. $e$ et $m$ sont la charge (considérée ici en valeur absolue) et la masse de l'électron.

Pour un ensemble d'électrons d'une couche $n$, on généralise ces résultats en considérant les nombres quantiques de spin total $S$ et de moment orbital total $L$ tel que les modules des moments cinétiques associés sont définis par :

$$
\sqrt{S(S+1)} \hbar, \quad \sqrt{L(L+1)} \hbar \text { et } \sqrt{J(J+1)} \hbar
$$

avec $\mathbf{J}=\mathbf{L}+\mathbf{S}$ et $|L-S|<J<L+S$.

On montre que le noyau atomique est également animé d'un mouvement de spin auquel on associe un moment cinétique $\mathbf{G}_{n}$ et un moment magnétique $\mathbf{M}_{n}$ tel que :

$$
\mathbf{M}_{n}=\gamma_{n} \mathbf{G}_{n} \quad \text { avec } \quad \gamma_{n}=\frac{Q}{M C}
$$

où $Q$ et $M$ sont la charge et la masse du noyau. Les signes des charges électroniques et nucléaires étant opposés on retiendra des relations (1) et (2), que $\gamma_{l}$ est négatif (donc $\mathbf{M}_{l}$ et $\mathbf{G}_{l}$ sont antiparallèles) et que $\gamma_{n}$ est positif (donc $\mathbf{M}_{n}$ et $\mathbf{G}_{n}$ sont parallèles). Par ailleurs on note que la différence entre masse électronique et nucléaire impose $\gamma_{n} \ll \gamma_{l}$.

Considérons maintenant l'évolution d'un moment magnétique soumis à un champ magnétique statique $H_{0}$ sans tenir compte pour le moment de la nature quantique et discrète du moment cinétique. Il est bien connu que l'action de $\mathbf{H}_{0}$ sur $\mathbf{M}$ se traduit par un couple mécanique $\left(\mathbf{M} \times \mathbf{H}_{0}\right)$ qui tend à aligner $\mathbf{M}$ suivant $\mathbf{H}_{0}$. Mais d'après ce qui précède on sait que $\mathbf{M}$ est un vecteur axial et non pas un vecteur polaire, de par son origine lié à un moment cinétique $\mathbf{G}$. Le moment magnétique possède donc les propriétés du gyroscope et doit ainsi se déplacer suivant une direction perpendiculaire à la fois à l'axe du girateur $\mathbf{G}$ et à la direction du champ perturbateur $H_{0}$. Cela entraîne le mouvement de précession autour de l'axe du champ à la fréquence de Larmor $\omega_{0}$. L'équation d'évolution est définie par le théorème du moment cinétique ( $=$ la dérivée par rapport au temps du moment cinétique est égale au couple extérieur appliqué) soit :

$$
\frac{\mathrm{d} \mathbf{G}}{\mathrm{d} t}=\text { couple }=\mathbf{M} \times \mathbf{H}_{0}
$$

ou en tenant compte des relations entre $\mathbf{G}$ et $\mathbf{M}$ :

$$
\frac{\mathrm{d} \mathbf{M}}{\mathrm{d} t}=\gamma\left(\mathbf{M} \times \mathbf{H}_{0}\right)
$$

où $\gamma$ est positif ou négatif suivant que $\mathbf{M}$ est un moment nucléaire ou électronique. On montre sans difficulté que cette équation d'évolution de l'aimantation possède une solution harmonique pour les composantes transverses $M_{x, y}$ de l'aimantation à la fréquence

$$
\omega_{0}=-\gamma H_{0} .
$$

A cause du signe opposé de leur facteur gyromagnétique, les moments magnétiques nucléaires et électroniques précessent respectivement suivant le sens d'une vis à gauche et à droite se déplaçant suivant la direction du champ magnétique comme indiqué sur les figures $3 a$ et $3 b$.

Pour mieux comprendre le couplage de $\mathbf{M}$ avec un rayonnement électromagnétique, il est intéressant d'analyser l'évolution de $\mathbf{M}$ non plus à partir du référentiel $x y z$ du laboratoire, mais à partir d'un référentiel $X Y Z$ tournant autour de la direction du champ statique $H_{0}$ à la vitesse angulaire $\omega$ (Fig. $3 c$ ).
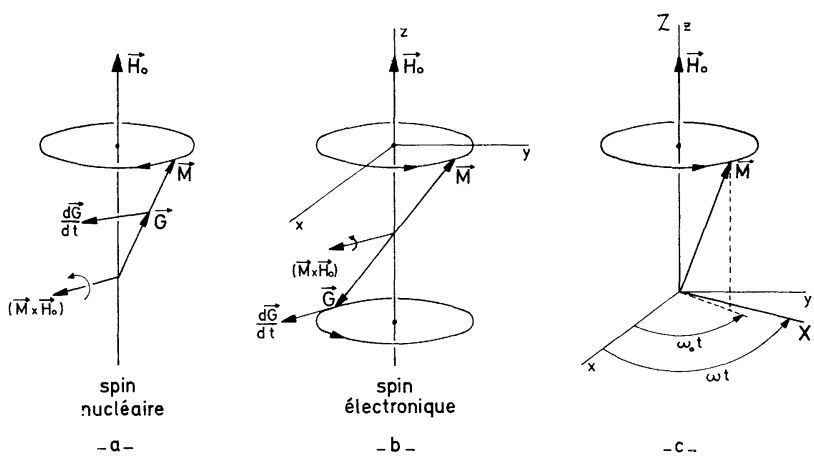

FIG. 3. 
Si on suppose $\omega<\omega_{0}$, un observateur placé dans le référentiel tournant voit $\mathbf{M}$ précesser non plus à la fréquence $\omega_{0}$ mais à une fréquence plus faible

$$
\omega^{\prime}=\omega_{0}-\omega .
$$

Tout se passe dans le système de coordonnées $X Y Z$ comme si $\mathbf{M}$ était soumis à un champ statique $\mathbf{H}^{\prime}$ plus faible. En transposant l'équation d'évolution 3 dans le référentiel tournant on montre aisément que la réduction apparente $H_{\omega}$ du champ statique est égale à $-\omega / \gamma$ tel que :

$$
H^{\prime}=H_{0}-H_{\omega}=H_{0}+\frac{\omega}{\gamma} \text {. }
$$

Si $\omega$ devient égal à $\omega_{0}$ le champ équivalent s'annule, tout mouvement de précession disparaît et $\mathbf{G}$ et $\mathbf{M}$ sont donc au repos dans le système tournant $X Y Z$.

2.2 EXCITATION RADIOFRÉQUENCE DE LA PRÉCESsion. - Résonance magnétique. Mouvement hors résonance. Amortissement de la résonance. Description classique.

Dans un modèle microscopique l'angle $\theta$ entre la direction $o z$ du champ $\mathbf{H}_{0}$ et le moment magnétique élémentaire est quantifié comme précisé par les nombres quantiques magnétiques $m_{L}, m_{S}$ ou $m_{J}$.

L'énergie $W$ du dipôle dépend donc de $\theta$, donc de $m_{J}$ (en prenant le cas d'un couplage LS par exemple) tel que

$$
W=-\mathbf{M H}_{0}=-g_{J} m_{J} \mu_{\mathbf{B}} H_{0} .
$$

A l'équilibre thermodynamique à la température $T$, les niveaux d'énergie les plus bas d'un ensemble de moments paramagnétiques sont plus occupés comme décrit par le facteur de Boltzmann $\exp \left(-W / k_{\mathrm{B}} T\right)$. Macroscopiquement on observe un moment résultant décrit dans la théorie du paramagnétisme par la fonction de Brillouin $B_{J}\left(W / k_{\mathrm{B}} T\right)$ qui représente un équilibre entre l'action de $H_{0}$ qui tend à ordonner les moments suivant $o z$ et l'action de l'agitation thermique qui tend à désorienter ces moments.

Macroscopiquement l'effet gyromagnétique correspond à la précession de ce moment résultant autour de $\mathbf{H}_{0}$ suivant un angle $\theta$ qui représente une moyenne de la direction des moments élémentaires. Ce mouvement n'est pas sans perte et $\theta$ diminue au cours de la précession. Nous analysons maintenant la possibilité d'entretenir ce mouvement de précession en considérant un moment macroscopique $\mathbf{M}$ soumis simultanément à un champ statique $\mathbf{H}_{0}$ et un champ magnétique oscillant $h(\omega)$ à la fréquence $\omega$ dans le plan xoy comme indiqué sur la figure 4 . Si $\mathbf{h}(\omega)$ est polarisé linéairement, il apparaît intuitivement que seule la composante circulaire tournant dans le même sens que la précession sera fortement couplée à l'aimantation. L'évolution de $\mathbf{M}$ dans ce cas est facile à comprendre si on se place dans un référentiel solidaire de $\mathbf{h}(\omega)$ (direction $O X$ sur la figure 4) et tournant avec la vitesse angulaire $\omega$ du champ radiofréquence.

Dans ce système de coordonnées tournantes $\mathbf{M}$ est soumis à un mouvement de précession de fréquence $\omega_{\text {eff }}$ autour d'un champ statique effectif

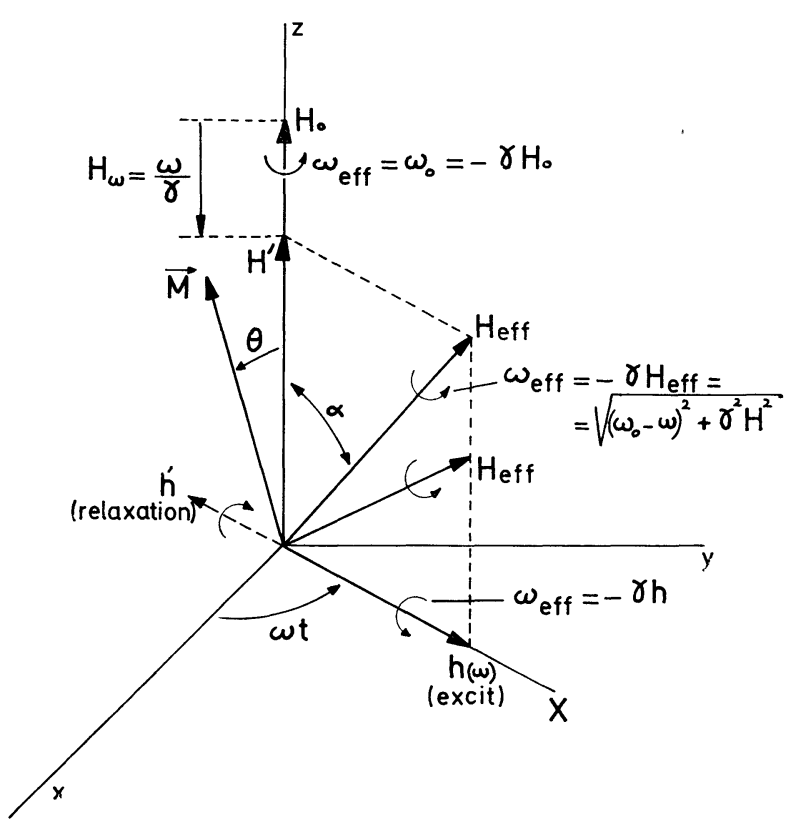

FIG. 4.

$\mathbf{H}_{\text {eff }}$ qui est la résultante géométrique de $\mathbf{h}$ et de $\mathbf{H}^{\prime}=\mathbf{H}_{0}-\mathbf{H}_{\omega}$ tel que :

$$
\omega_{\text {eff }}=-\gamma H_{\text {eff }}=\left[\left(\omega_{0}-\omega\right)^{2}+\gamma^{2} h^{2}\right]^{1 / 2} .
$$

L'angle $\alpha$ entre $H_{\text {eff }}$ et l'axe $O Z$ est d'autant plus important que la réduction apparente $H_{\omega}$ du champ appliqué est élevée, donc que $\omega$ tend vers la fréquence de Larmor $\omega_{0}=-\gamma H_{0}$. Si $\omega=\omega_{0}, \alpha=\pi / 2$ et dans le référentiel tournant le mouvement de l'aimantation se réduit à une précession autour du champ magnétique $\mathbf{h}$ avec une vitesse angulaire

$$
\omega_{\mathrm{eff}}=\frac{\mathrm{d} \theta}{\mathrm{d} t}=-\gamma h .
$$

Si on fait abstraction pour le moment de l'amortissement du système, l'angle $\theta$ augmente dans le temps comme :

$$
\theta=-\gamma h t .
$$

C'est le phénomène de résonance magnétique décrit dans le référentiel tournant. Dans le référentiel du laboratoire, $\mathbf{M}$ évolue suivant une spirale située sur une sphère comme indiqué sur la figure 5 . L'angle polaire $\theta$ augmente d'autant plus vite que l'amplitude $h$ de l'excitation oscillante est élevée. En pratique l'action du pompage radiofréquence $\boldsymbol{h}$ est limitée par les pertes du mouvement gyromagnétique et à la résonance $\theta$ atteint une valeur stationnaire bien précise. 


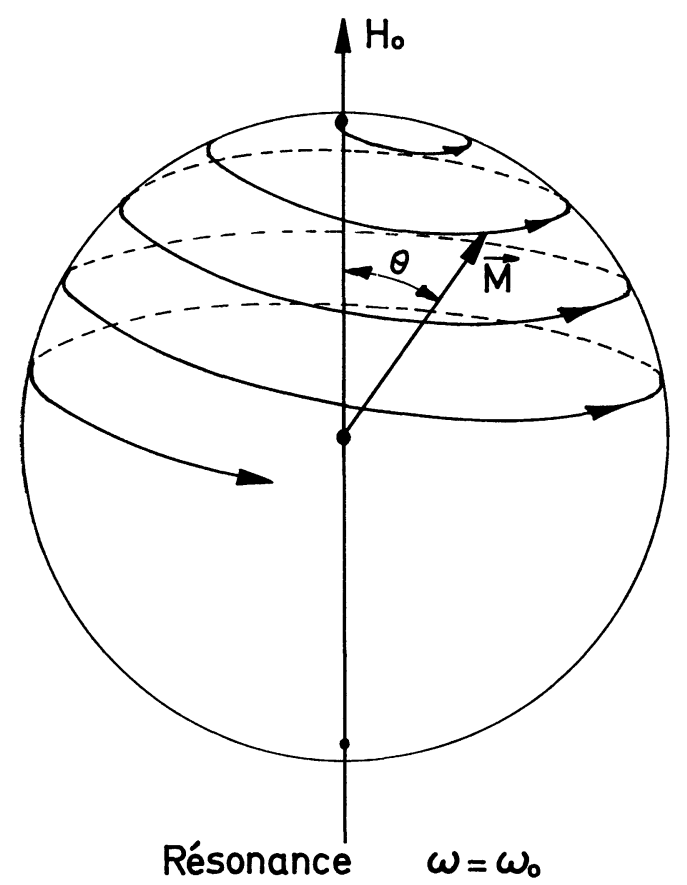

FIG. 5.

2.2.1 Mouvement hors résonance. - Si $\omega \neq \omega_{0}$ le mouvement de $\mathbf{M}$ est une simple précession autour de $\mathbf{H}_{\text {eff }}$ dans le référentiel tournant. Dans le référentiel $\mathrm{du}$ laboratoire il faut superposer ce mouvement à celui d'une seconde précession à la fréquence $\omega$ autour de $\mathbf{H}_{0}$. Suivant que $\omega$ est inférieur ou supérieur à $\omega_{0}$ on obtient les évolutions décrites par les figures 6 et 7 où la valeur de $\theta$ a été exagérée, car cet angle est non négligeable uniquement à la résonance. Hors résonance il existe donc un mouvement contraint à la fréquence $\omega$ de l'excitation radiofréquence.

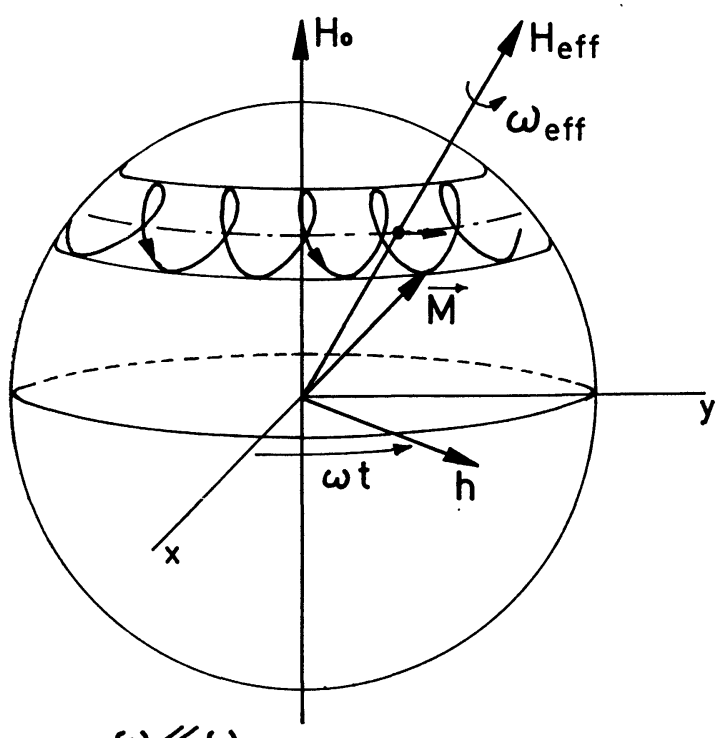

$\omega \ll \omega_{\circ}$

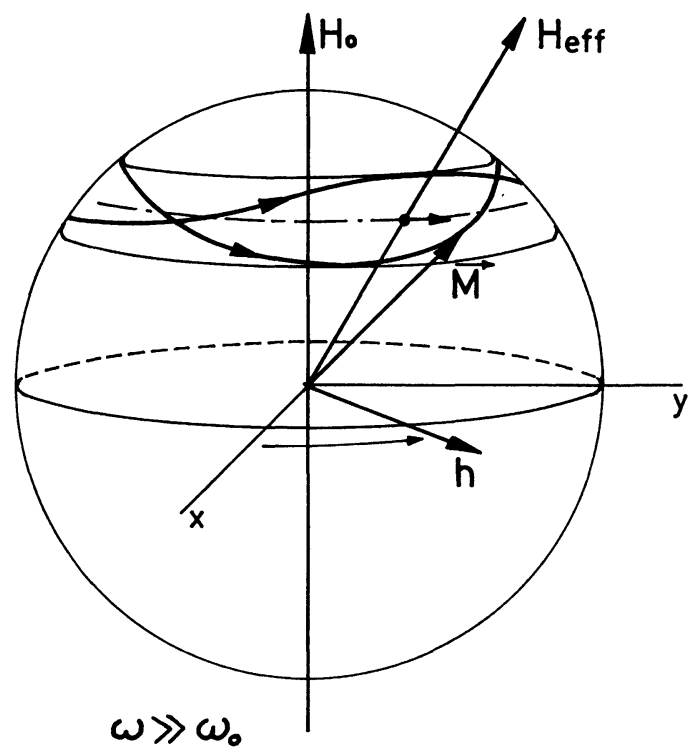

FIG. 7.

2.2.2 Amortissement de la résonance. Relaxation magnétique. - Pendant la résonance, l'énergie du moment magnétique augmente au détriment de l'excitation r. f. d'autant plus que l'angle $\theta$ de précession est élevé. En fait cet angle ne croît pas indéfiniment car $\mathbf{M}$ est non pas isolé mais couplé à d'autres systèmes auxquels il transfère son énergie plus ou moins rapidement. Dès lors, si un certain instant après excitation de la résonance on coupe brusquement cette excitation, le moment magnétique macroscopique tend à s'aligner suivant $\mathbf{H}_{0}$ après un mouvement de spirale plus ou moins complexe fonction des processus microscopiques de transfert énergétique. Dans de nombreux cas le temps d'amortissement ou temps de relaxation peut se décomposer en 2 temps $T_{2}$ et $T_{1}$ qui décrivent l'amortissement des composantes transverse $M_{x, y}$ et longitudinale $M_{z}$ de l'aimantation, comme décrit sur la figure 8 . Une telle décomposition

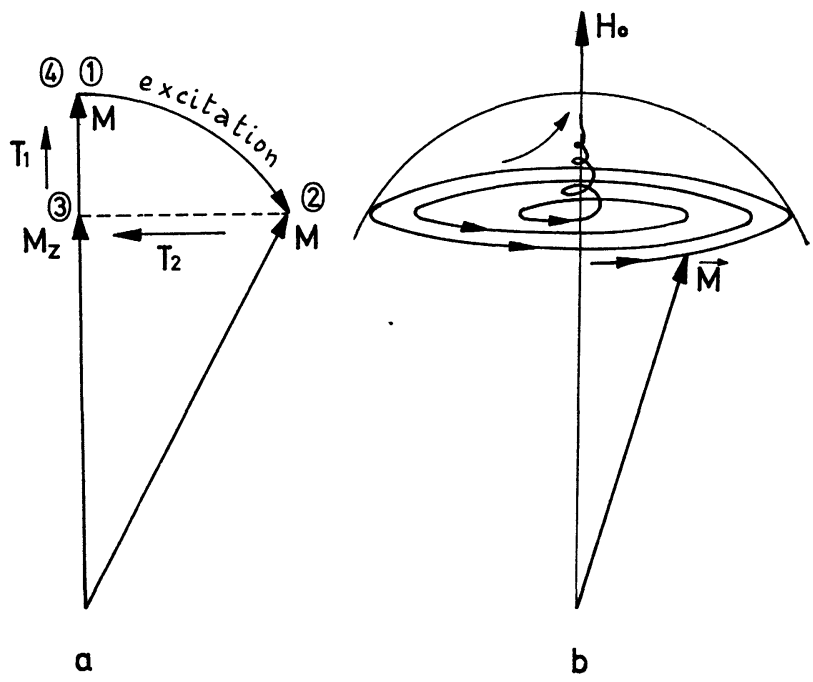

Fig. 8.

FIG. 6.

8 
est possible car la plupart du temps $T_{2}$ est très court devant $T_{1}$. La composante $M_{z}$ de l'aimantation totale reste constante durant $T_{2}$ qui représente la relaxation spin-spin ou relaxation transverse pendant laquelle les spins élémentaires perdent dans le plan xoy leur cohérence de phase spatiale (donc $M_{x}$ et $M_{y} \rightarrow 0$ ). Ces processus sont induits par l'interaction dipôledipôle où des termes du type $S_{\mathrm{i}}^{+} S_{\mathrm{j}}^{-}$représentent le transfert d'une excitation magnétique du site $\mathrm{i}$ vers le site j (Fig. 9a). Un tel transfert peut se faire à grande distance puisque l'interaction dipolaire est à longue portée.
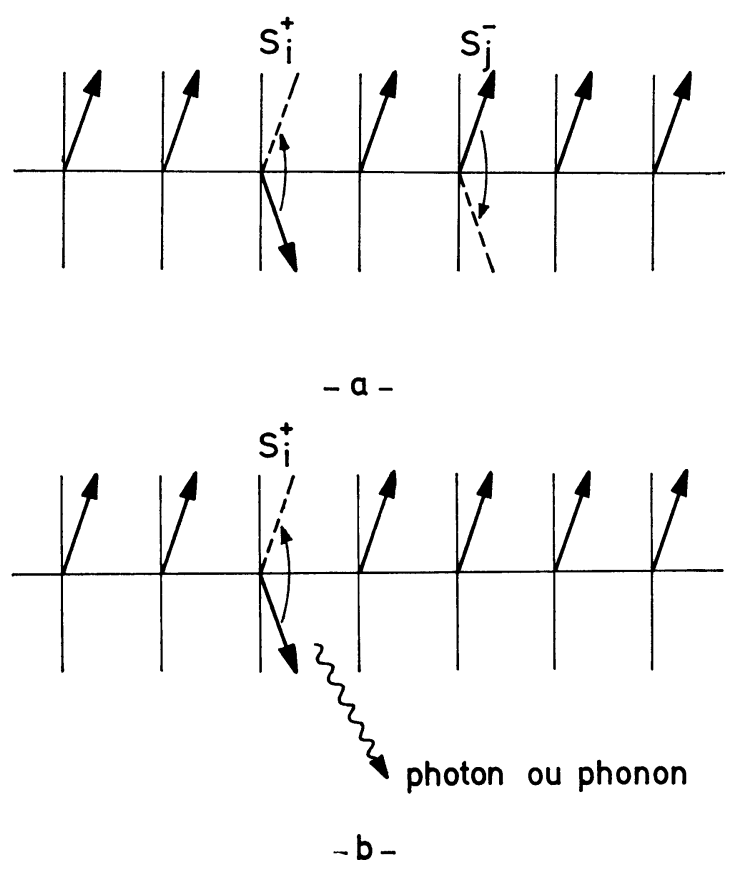

FIG. 9.

Dans un deuxième temps $T_{1}, M_{z}$ tend vers la valeur initiale de l'aimantation $\left(M_{z} \rightarrow M\right)$ au repos, ce qui se traduit par un transfert de l'énergie d'excitation magnétique vers d'autres systèmes non magnétiques. Cette relaxation longitudinale peut être associée à des processus soit radiatifs (transferts spin $\rightarrow$ photon) soit non radiatifs (transferts spin-réseau ou spinphonon) (Fig. 9b). L'évolution de l'aimantation macroscopique $\mathbf{M}$ en présence d'une excitation $r$. $f$. $h(\omega)$ et de processus d'amortissement est décrite à partir de la relation 3 par l'équation suivante :

$\frac{1}{\gamma} \frac{\mathrm{d} \mathbf{M}}{\mathrm{d} t}=\left[\mathbf{M} \times\left(\mathbf{H}_{0}+\mathbf{h}\right)\right]+\frac{\alpha}{|M|}\left[\mathbf{M} \times\left(\mathbf{M} \times \mathbf{H}_{\mathrm{t}}\right)\right]$

où $H_{\mathrm{t}}$ est le champ total et $\alpha$ est un facteur d'amortissement phénoménologique introduit par Landau et Lifshitz. On vérifie que l'expression vectorielle de ce terme d'amortissement tend à diminuer l'angle du cône de précession (Fig. 10). Des termes d'amortissement autres que celui de Landau ont été proposés pour mieux représenter certains cas particuliers de relaxation. Nous citons pour mémoire celui de Bloem-

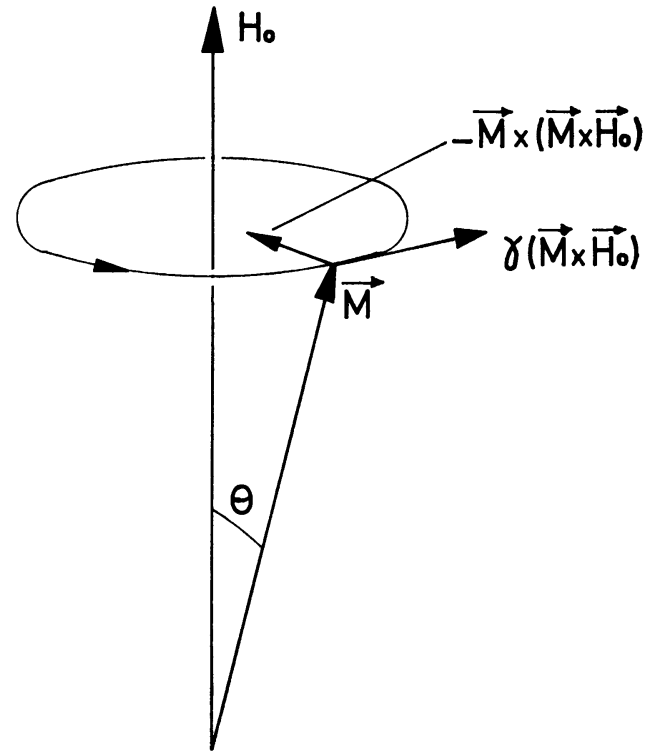

FIG. 10

bergen qui a l'avantage de séparer les relaxations longitudinales et transverses tel que :

$$
\begin{aligned}
\frac{\mathrm{d} M_{x, y}}{\mathrm{~d} t} & =\gamma\left[\mathbf{M} \times\left(\mathbf{H}_{0}+\mathbf{h}\right)\right]_{x, y}-\frac{M_{x, y}}{2 T_{2}} \\
\frac{\mathrm{d} M_{z}}{\mathrm{~d} t} & =\gamma\left[\mathbf{M} \times\left(\mathbf{H}_{0}+\mathbf{h}\right)\right]_{z}-\frac{M_{z}-M_{0}}{2 T_{1}}
\end{aligned}
$$

où $M_{0}$ est l'aimantation totale au repos.

La réponse $\mathbf{m}$ de système magnétique à une excitation harmonique $\mathbf{h}$ polarisée linéairement dans le plan xoy est décrite par la susceptibilité dynamique $\chi$ qui comprend deux valeurs propres $\chi_{+}$et $\chi_{-}$ associées aux deux composantes contrarotatives droite et gauche $h_{+}$et $h_{-}$du champ excitateur dans le plan xoy tel que :

$$
\left|\begin{array}{l}
m_{+} \\
m_{-}
\end{array}\right|=\left|\begin{array}{cc}
\chi_{+} & 0 \\
0 & \chi_{-}
\end{array}\right| \cdot\left|\begin{array}{l}
h_{+} \\
h_{-}
\end{array}\right|
$$

avec

$$
\left\{\begin{aligned}
m_{ \pm} & =M_{x} \pm \mathrm{i} M_{y} \\
h_{ \pm} & =h_{x} \pm \mathrm{i} h_{y}
\end{aligned}\right\}
$$

$m_{+}$étant le mode propre tournant dans le plan xoy dans le même sens que la précession de Larmor, son amplitude devient importante à la résonance, $m_{-}$tourne dans le sens opposé, aussi son amplitude reste-t-elle toujours très faible. $m_{+}$et $m_{-}$sont appelés modes résonnant et anti-résonnant respectivement. Les expressions de $\chi_{ \pm}$sont complexes et peuvent être déduites de l'équation d'évolution 9 sans difficulté :

$$
\begin{aligned}
& \chi_{ \pm}=\chi_{ \pm}^{\prime} \pm \mathrm{i} \chi_{ \pm}^{\prime \prime}=\frac{\omega_{\mathrm{m}}}{\omega_{0} \mp \omega(1 \mp \mathrm{j} \alpha)} \\
& \chi_{ \pm}^{\prime}=\frac{\omega_{\mathrm{m}}\left(\omega_{0} \mp \omega\right)}{\left(\omega_{0} \mp \omega\right)^{2}+\alpha^{2} \omega^{2}} \\
& \chi_{ \pm}^{\prime \prime}=\frac{\alpha \omega \omega_{\mathrm{m}}}{\left(\omega_{0} \mp \omega\right)^{2}+\alpha^{2} \omega^{2}}
\end{aligned}
$$


avec $\omega_{\mathrm{m}}=\gamma 4 \pi M_{0}$. $\chi^{\prime}$ et $\chi^{\prime \prime}$ représentent les composantes dispersives et d'absorption du mouvement gyromagnétique qui présentent une anomalie au voisinage de la résonance. L'amplitude de l'aimantation harmonique est proportionnelle à $\chi_{+}^{\prime \prime}$ qui est maximale à la résonance $\left(\chi_{+}^{\prime \prime}=\omega_{\mathrm{m}} / \alpha \omega_{0}\right.$, cf. éq. (12)). $\chi_{+}^{\prime \prime}$ permet donc d'évaluer à la fois l'énergie magnétique stockée par le mouvement gyromagnétique et les pertes de ce mouvement $\left(\chi_{+}^{\prime \prime}\right.$ est inversement proportionnel à $\alpha$ ). On vérifie à partir des équations 12 que la largeur à mi-hauteur des courbes $\chi_{+}^{\prime \prime}=f(\omega)$ où $\chi_{+}^{\prime \prime}=f\left(H_{0}\right)$, soient $\Delta \omega$ ou $\Delta H$, est directement proportionnelle au facteur d'amortissement $\alpha$ : $\Delta \omega=2 \alpha \omega_{0}$ ou $\Delta H=2 \alpha H_{0}$ (Fig. 11).

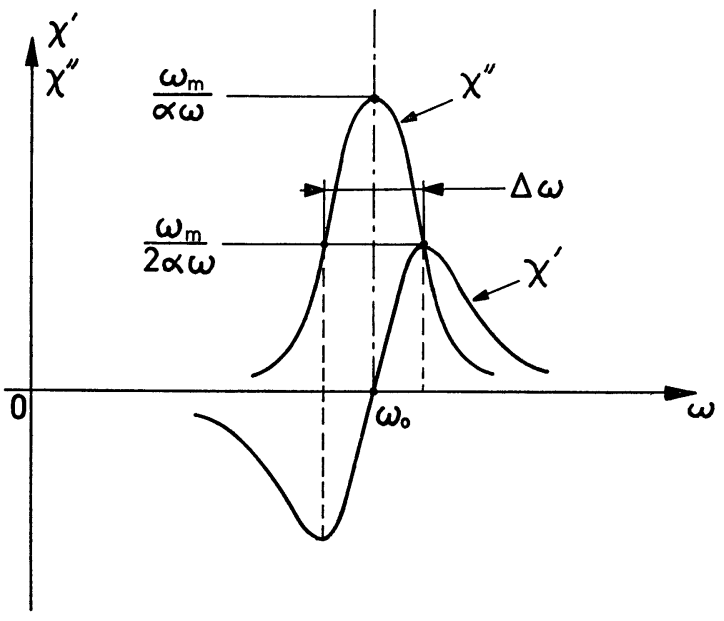

FIG. 11.

2.3 Description QuANTIQUe DE L'EXCITATION ET DE L'AMORTISSEMENT DU MOUVEMENT GYROMAGNÉTIQUE. IMPORTANCE DES TRANSITIONS RÉELLES ET VIRTUELLES. Nous pouvons donner une description quantique simple et intuitive de l'excitation résonnante du mouvement gyromagnétique en appliquant le schéma macroscopique discuté plus haut au modèle vectoriel de l'atome (Fig. 12a). Lorsque la condition de résonance est satisfaite $\omega=\gamma H_{0}=\omega_{0}$, on peut considérer que la précession du spin $\mathbf{S}$ (ou du moment magnétique associé) autour du champ excitateur h (l'observateur se situe dans le référentiel tournant à la vitesse

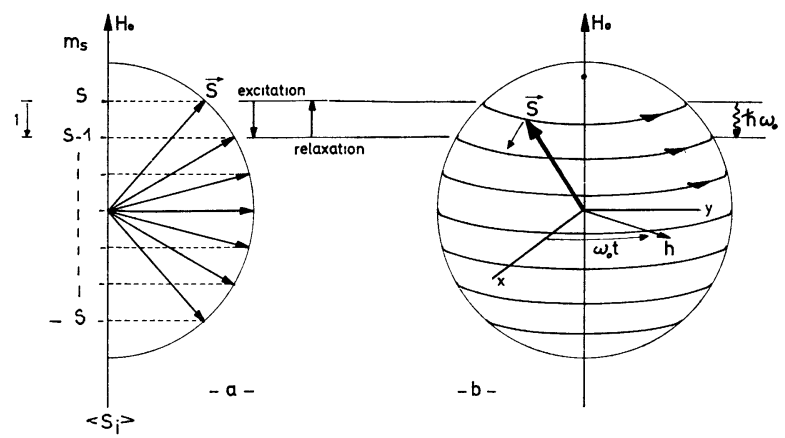

FIG. 12. angulaire $\omega_{0}$ ) s'effectue de façon discontinue puisque les valeurs de $m_{S}$ sont quantifiées. Toutes les autres propriétés décrites dans le modèle macroscopique restent applicables. Dès lors on considère que le rôle du champ excitateur est double. Si $\omega=\omega_{0}$, h opère d'une part la mise en phase spatiale des spins sur les différents cônes de précession, et d'autre part induit des transitions de l'état de base vers les états excités $\left(m_{S}=S \rightarrow m_{S}=S-1\right.$ sur la figure $\left.12 a\right)$.

L'énergie du photon radiofréquence absorbe $\hbar \omega$ est égale à l'énergie séparant deux états magnétiques voisins soit :

$$
\hbar \omega=\Delta W=g \mu_{\mathrm{B}} H_{0}=\hbar \gamma H_{0}=\hbar \omega_{0} .
$$

Si $\omega \neq \omega_{0}$, il y a toujours mise en phase spatiale des spins, mais les processus d'absorption des photons $r$. $f$. permettant les transitions entre états magnétiques ne sont plus autorisés de façon réelle comme à la résonance.

2.3.1 Importance des transitions réelles et virtuelles. - Une interaction spin-photon hors résonance apparaît encore et peut être décrite de manière simple à partir de la notion de transition virtuelle qui n'est autre qu'un effet tunnel de photon. A cet effet, considérons la levée de dégénérescence par $\mathbf{H}_{0}$ des états $\left|J, m_{J}\right\rangle$ d'un ion magnétique de nombre quantique $J=2$ par exemple (Fig. 13). L'état de base correspond

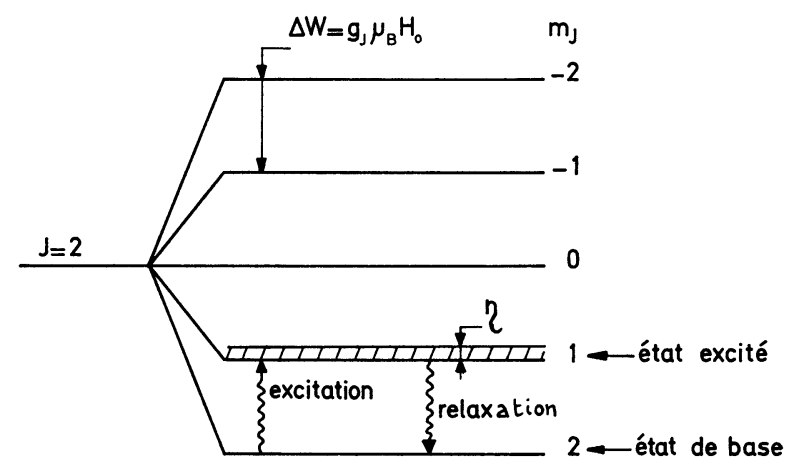

FIG. 13.

à $m_{J}=2$ et le premier état excité à $m_{J}=1$. Il est connu que toute variation de population d'un état initial $\mid \mathrm{i}>$ excité (par relaxation ou émission de 1 photon) entraîne un élargissement $\eta_{i}$ (largeur de raie naturelle) est un déplacement $\delta_{\mathbf{i}}$ de cet état. $\eta_{\mathrm{i}}$ est inversement proportionnel à la durée de vie $T_{\mathbf{i}}$ du niveau initial en accord avec la relation d'incertitude de Heisenberg : $\Delta E=\hbar \eta_{\mathrm{i}}=\hbar / T_{\mathrm{i}}$. L'absorption ou l'émission de un photon est possible si son énergie $\hbar \omega$ est exactement égale à la séparation énergétique entre le niveau initial $|\mathrm{i}\rangle$ et final $|\mathrm{f}\rangle$ d'énergie $E_{\mathrm{i}}$ et $E_{\mathrm{f}}$ à la largeur près $\left(\eta_{\mathrm{i}}+\eta_{\mathrm{f}}\right)$ de ces niveaux. L'absorption ou l'émission d'un photon constitue une transition réelle.

Les transitions réelles ne représentent qu'une partie des interactions spin-photon possibles car en fait il 
faut envisager deux cas : Si $\left|\hbar \omega-\hbar \omega_{\mathrm{fi}}\right|<\hbar\left(\eta_{\mathrm{i}}+\eta_{\mathrm{f}}\right)$ (avec $\hbar \omega_{\mathrm{fi}}=E_{\mathrm{f}}-E_{\mathrm{i}}$ ), le photon peut être effectivement absorbé et il y a transition réelle.

Si par contre $\left|\hbar \omega-\hbar \omega_{\mathrm{fi}}\right|>\hbar\left(\eta_{\mathrm{i}}+\eta_{\mathrm{f}}\right)$, il y a un excès (ou un défaut) d'énergie $\Delta=\hbar\left(\omega-\omega_{\mathrm{fi}}\right)$ qui fait que le photon ne peut plus être réellement absorbé. Une interaction photon-atome est encore possible dans ce cas, mais sous forme de transition virtuelle dans laquelle le photon est «capté » par l'atome pendant un temps $\Delta t$ extrêmement court puis réémis. Ce temps de capture, qui, là encore, est défini par la relation d'incertitude de Heisenberg: $\Delta t=\hbar / \Delta$, est d'autant plus important que l'écart énergétique, $\Delta$ est faible jusqu'au cas limite $\Delta<\hbar\left(\eta_{\mathrm{i}}+\eta_{\mathrm{f}}\right)$ où l'on réalise à nouveau une transition réelle. Cette capture est fondamentalement identique à "l'effet tunnel » où $\Delta$ n'est autre que la barrière de potentiel que le système global (atome + photon) doit franchir. La transition virtuelle existe réellement mais son appellation provient de sa durée extrêmement brève $\left(\Delta t \simeq 10^{-16} \mathrm{~s}\right.$ dans certains cas) comparée aux temps de relaxation $\left(T=10^{-5}\right.$ à $\left.10^{-8} \mathrm{~s}\right)$ associés aux transitions réelles. Tous les phénomènes de dispersion et d'absorption sont décrits microscopiquement à partir des transitions virtuelles et réelles. Il en sera ainsi pour les biréfringence et dichroïsme magnétiques circulaires, dans les bandes $r$. f. et optique, de la résonance magnétique, etc...

Puisqu'à tous phénomènes d'absorption est associé un phénomène de dispersion, les transitions ne peuvent être purement réelles ou purement virtuelles que dans certains cas limites, comme indiqué sur la figure 14 où la dispersion et l'absorption associées

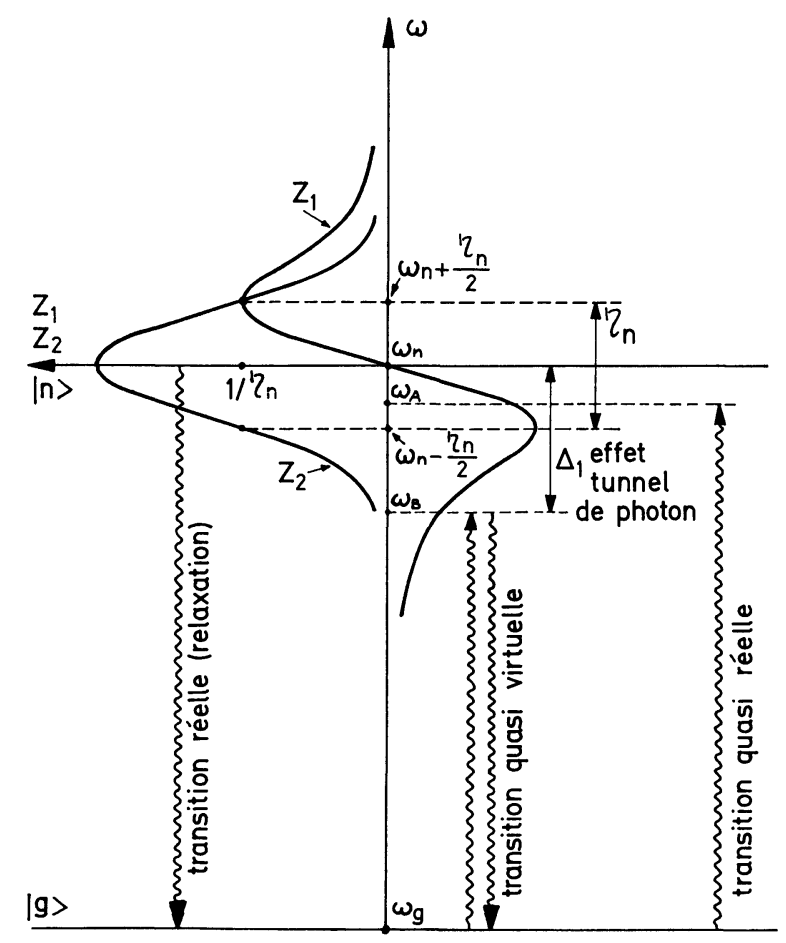

FIG. 14. à la relaxation du niveau excité $\mid \mathrm{n}>$ correspondent aux parties réelle et imaginaire de la fonction dzéta de Dirac (| g > est l'état de base) :

$$
\begin{aligned}
& \zeta\left(\omega_{\mathrm{ng}}-\omega_{q}\right)= \\
& =z_{1}-\mathrm{i} z_{2}=\lim _{\eta_{\mathrm{n}} \rightarrow 0} \frac{1}{\left(\omega_{\mathrm{ng}}-\omega_{q}\right)+\mathrm{i}\left(\eta_{\mathrm{n}} / 2\right)} \\
& z_{1}=\frac{P}{\omega_{\mathrm{ng}}-\omega_{q}}=\lim _{\eta_{\mathrm{n}} \rightarrow 0} \frac{\omega_{\mathrm{ng}}-\omega_{q}}{\left(\omega_{\mathrm{ng}}-\omega_{q}\right)^{2}+\left(\eta_{\mathrm{n}}^{2} / 4\right)} \\
& z_{2}=\pi \delta\left(\omega_{\mathrm{ng}}-\omega_{q}\right)=\lim _{\eta_{\mathrm{n}} \rightarrow 0} \frac{\eta_{\mathrm{n}}}{2\left[\left(\omega_{\mathrm{ng}}-\omega_{q}\right)^{2}+\left(\eta_{\mathrm{n}}^{2} / 4\right)\right]}
\end{aligned}
$$

Loin d'une raie d'absorption, la dispersion décroit moins vite $\left(1 / \omega_{\mathrm{ng}}-\omega\right)$ que l'absorption $\left(\eta /\left(\omega_{\mathrm{ng}}-\omega\right)^{2}\right)$, ce qui explique que les effets dispersifs peuvent encore apparaître en dehors des zones d'absorption. Le niveau $|\mathrm{n}\rangle$ étant amorti, son excitation par un rayonnement extérieur est à caractère essentiellement d'absorption si $\left|\omega_{\mathrm{ng}}-\omega\right|<\eta_{\mathrm{n}} / 2$, ou à caractère essentiellement dispersif si $\left|\omega_{\mathrm{ng}}-\omega\right|>\eta_{\mathrm{n}} / 2$. Dans le premier cas la transition est strictement réelle si $\omega_{\mathrm{ng}}-\omega=0$ et quasi réelle si $\left|\omega_{\mathrm{ng}}-\omega\right|<\eta_{\mathrm{n}} / 2$. Dans le second cas la transition est quasi virtuelle. Le gap $\Delta_{1}$ produit un effet tunnel de photon qui se traduit par la diffusion du photon par l'atome. La diffusion spin-photon est élastique dans les biréfringences magnétiques circulaire et linéaire et inélastique dans les effets Raman de spin ou de magnon. La figure 14 montre que si une séparation entre les transitions réelles et virtuelles ne peut être établie de manière absolue, il est toutefois possible de définir une région d'interaction à caractère quasi réel (zone hachurée de largeur $\Delta \omega_{\mathrm{n}}=\eta_{\mathrm{n}}$ ) et une région à caractère quasi virtuel. Ces notions sont fondamentales pour la description microscopique des biréfringence et dichroïsme magnétique, et des phénomènes de résonance magnétique.

2.3.2 Termes énergétiques d'interaction. - Considérons un dipôle magnétique $\mathbf{M}$ en interaction avec un champ magnétique statique $\mathbf{H}_{0}$ dirigé suivant $O z$ et un champ r. f. h de polarisation et de direction quelconque. L'interaction est décrite par :

$$
\mathscr{H}_{\mathrm{int}}=-\mathbf{M} \cdot\left(\mathbf{H}_{0}+\mathbf{h}\right)=-M_{z} H_{0}-\mathbf{M} \cdot \mathbf{h}
$$

où le second terme est une perturbation harmonique qui peut induire des transitions entre les niveaux non dégénérés par la présence du champ statique $H_{0}$. h est une fonction sinusoïdale $\left(\sim \cos \omega_{q} t\right)$ qui peut toujours se décomposer dans l'espace complexe en deux composantes contrarotatives $h^{+}$et $h^{-}$telles que $\mathbf{h}\left(\omega_{q}\right)=\mathbf{h} \cos \omega_{q} t=\mathbf{h}^{+} \mathrm{e}^{-i \omega} q^{t}+\mathbf{h}^{-} \mathrm{e}^{i \omega} q^{t}$. Il en est de même pour le dipôle macroscopique $\mathbf{M}$ tel que :

$$
\begin{aligned}
\mathscr{H}_{\mathrm{int}}= & -M_{z} H_{0}-\left(M^{+}+M^{-}\right)\left(h^{+}+h^{-}\right) \\
=-M_{z} H_{0}-\left(M^{+} h^{-}\right. & +M^{+} h^{+}+ \\
& \left.+M^{-} h^{+}+M^{-} h^{-}\right) .
\end{aligned}
$$


Une description microscopique est donnée, dans le cas d'un magnétisme de spin par:

$$
\begin{gathered}
\mathscr{H}_{\mathrm{int}}= \\
=-\gamma S_{z} H_{0}-\gamma\left(S^{+} h^{-}+S^{+} h^{+}+S^{-} h^{+}+S^{-} h^{-}\right) .
\end{gathered}
$$

Il est intéressant d'effectuer un tel développement dans l'espace complexe car les composantes classiques $h^{+}$et $h^{-}$du rayonnement deviennent en mécanique quantique les opérateurs de destruction et de création de photon respectivement, tandis que les opérateurs $S^{+}$et $S^{-}$transforment un état $\mid S, m_{S}>$ tel que :

$$
\begin{aligned}
& S^{ \pm} \mid S m_{S}>= \\
& \quad=\sqrt{S(S+1)-m_{S}\left(m_{S} \pm 1\right)} \cdot \mid S, m_{S} \pm 1>
\end{aligned}
$$

Ces propriétés, alliées aux notions de transitions réelles et virtuelles discutées plus haut, permettent de comprendre la nature physique des quatre termes énergétiques harmoniques contenus dans l'Hamiltonien 14. Considérons en effet un état initial

$$
|\mathrm{i}\rangle=\left|S=2, m_{S}=+1\right\rangle
$$

(Fig. 15). L'opérateur $S^{+}$induit une transition du niveau $m_{S}=+1$ vers le niveau $m_{S}=+2$, d'énergie plus basse, soit par émission de un photon (transition $\mathrm{n}^{0} 1, S^{+} h^{-}$), soit par absorption de un photon (terme $S^{+} h^{+}$). Si la transition 1 peut être réelle et résonnante (gap $\left.\Delta_{1}=0\right)$ ou virtuelle $\left(\Delta_{1} \neq 0\right)$, la transition 2 est toujours virtuelle (gap $\Delta_{2} \neq 0$ ).

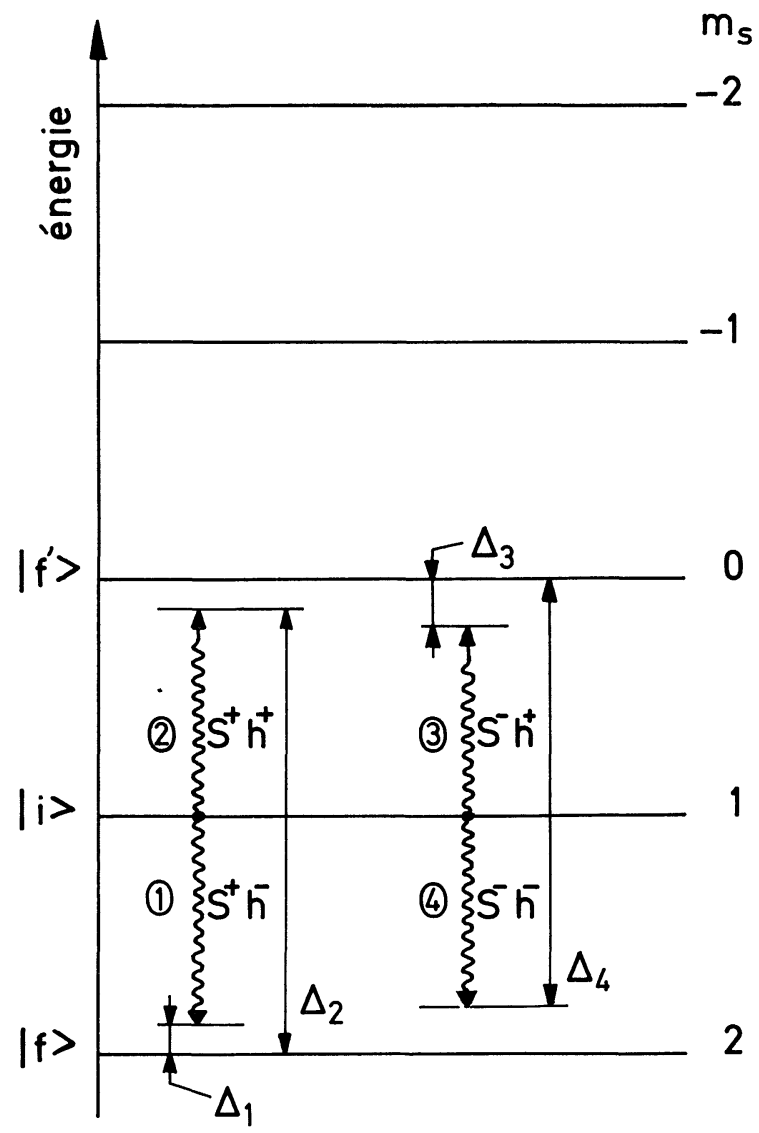

Fig. 15.

REVUE DE PHYSIOUE APPLIOUÉE. - T. 9 , No 5, SEPTEMBRE 1974
Cette transition sera donc en général faible. De la même façon, les termes $S^{-} h^{+}$et $S^{-} h^{-}$connectent l'état initial $m_{S}=+1$ à un état final $m_{S}=0$ par des transitions ou réelle $\left(S^{-} h^{+}\right)$du type absorption ou virtuelle $S^{-} h^{-}$par émission virtuelle de un photon (gap $\Delta_{4} \neq 0$ ). En général, la plupart des transitions virtuelles sont ignorées, ce qui explique la difficulté de compréhension de certaines analyses théoriques des interactions rayonnement-matière. Nous retrouverons ces propriétés dans l'étude des propriétés optiques des milieux magnétiques.

2.4 RÉSONANCE EN RÉGIME IMPULSIONNEL : ÉCHO DE SPIN. - Nous avons vu (section 2.2) qu'à la résonance le dipôle magnétique $\mathbf{M}$ subit dans le référentiel tournant $X Y Z$ (Fig. 4) une précession $\omega_{\text {eff }}=-\gamma h$ autour du champ r. f. ce qui se traduit par une ouverture progressive dans le temps de l'angle polaire $\theta=-\gamma h t$. Cet angle peut atteindre des valeurs importantes de l'ordre de $\pi / 2$ par exemple après un temps $t_{1}$ défini par :

$$
t_{1}=\frac{\pi}{2} \cdot \frac{1}{-\gamma h} \text {. }
$$

En pratique ceci n'est pas possible en régime stationnaire car les processus de relaxation limitent $\theta$ à des valeurs très faibles. On peut toutefois y parvenir en régime transitoire en appliquant une impulsion de durée inférieure aux temps de relaxation $T_{1}, T_{2}$ et d'amplitude $h$ telle que $t_{1} \ll T_{1}, T_{2}$. La mesure des temps de relaxation par la technique d'échos de spin utilise un tel artifice où l'application d'une première impulsion dite de $90^{\circ}$ produit une précession en phase des spins dans le plan équatorial (Fig. 16).

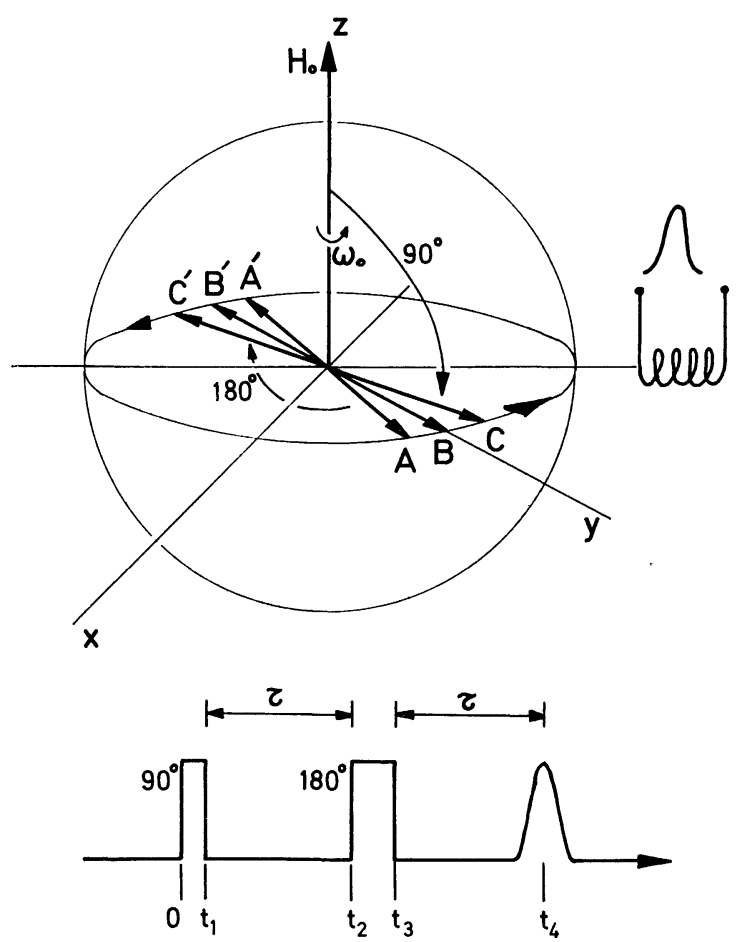

FIG. 16. 
Au temps $t=t_{1}$ l'impulsion disparaît et les spins perdent progressivement leur cohérence de phase par suite de transitions individuelles de relaxation et $\theta$ commence à décroître au cours d'un mouvement de précession autour de $H_{0}$. Si après un temps $\tau$ tel que $t_{1}<\tau<T_{1}, T_{2}$, on applique une seconde impulsion dite de $180^{\circ}(\theta \rightarrow \theta+\pi)$, les spins subissent un renversement complet de $\pi$ par rapport à l'axe tournant $O X$. Conséquences : Les spins qui étaient en avance de phase parce qu'ils précessaient légèrement plus vite, se retrouvent après renversement en retard de phase mais évoluant toujours plus rapidement que les autres spins. On prévoit donc qu'un temps $\tau$ après la seconde impulsion, tous les spins se retrouvent à nouveau en cohérence de phase avant de se disperser définitivement. Pendant le bref instant de remise en cohérence de phase une aimantation transverse importante peut induire dans une bobine d'axe perpendiculaire à $\mathbf{H}_{0}$ un signal magnétique ou écho de spin dont les caractéristiques sont précieuses pour déterminer par exemple les champs magnétiques internes ou les temps de relaxation $T_{1}$ et $T_{2}$ des spins électroniques et nucléaires.

A titre d'exemple, la durée d'une impulsion de $\pi / 2$ dans une expérience d'écho de spin électronique disposant d'une excitation $h=1$ Oe et $f=10 \mathrm{GHz}$ est égale à $t_{1}=90 \mathrm{~ns}$, au bout de laquelle les spins ont subi $t_{1} / T=900$ cycles de précession. Il est nécessaire d'utiliser des impulsions très brèves lorsque les temps de relaxation sont courts, ce qui nécessite l'emploi de sources $r$. f. de plusieurs kilowatts dans certains cas.

2.5 BASCUlEMENT DE SPIN. Evolution NON ADIABATIQUE. SPIN-FLIP. - Nous examinons maintenant l'évolution des amplitudes «spin-up» $\left(m_{s}=+\frac{1}{2}\right)$ et « spin down » $\left(m_{s}=-\frac{1}{2}\right)$ d'une particule de spin $\frac{1}{2}$ qui étant initialement alignée par un champ continu suivant la direction $+O z$, voit brusquement la direction de ce champ changer de direction. Un cas pratique correspond à la variation de l'angle polaire $\theta$ du champ magnétique de 0 à $\pi$ (Fig. 17). La résonance magnétique discutée plus haut est un cas particulier de ce problème puisque dans le référentiel tournant on observe un spin-flip induit par l'application du champ r. f. perpendiculairement au champ statique. Nous avons vu que ce basculement du dipôle magnétique est particulièrement efficace en utilisant les impulsions $\pi / 2$ et $\pi$ en technique d'écho de spin. Un second exemple correspond au passage adiabatique ou non adiabatique d'un flux de Iparticules de spin $S$ à travers une région de champ magnétique statique dont la direction varie progressivement au cours du déplacement des particules. Enfin un troisième exemple est celui de l'évolution d'un ensemble de dipôle magnétique soumis à un champ magnétique dont on inverse brusquement la direction. Ce dernier cas est étroitement associé au temps de basculement des cycles d'hystérésis de l'aimantation dans les maté-

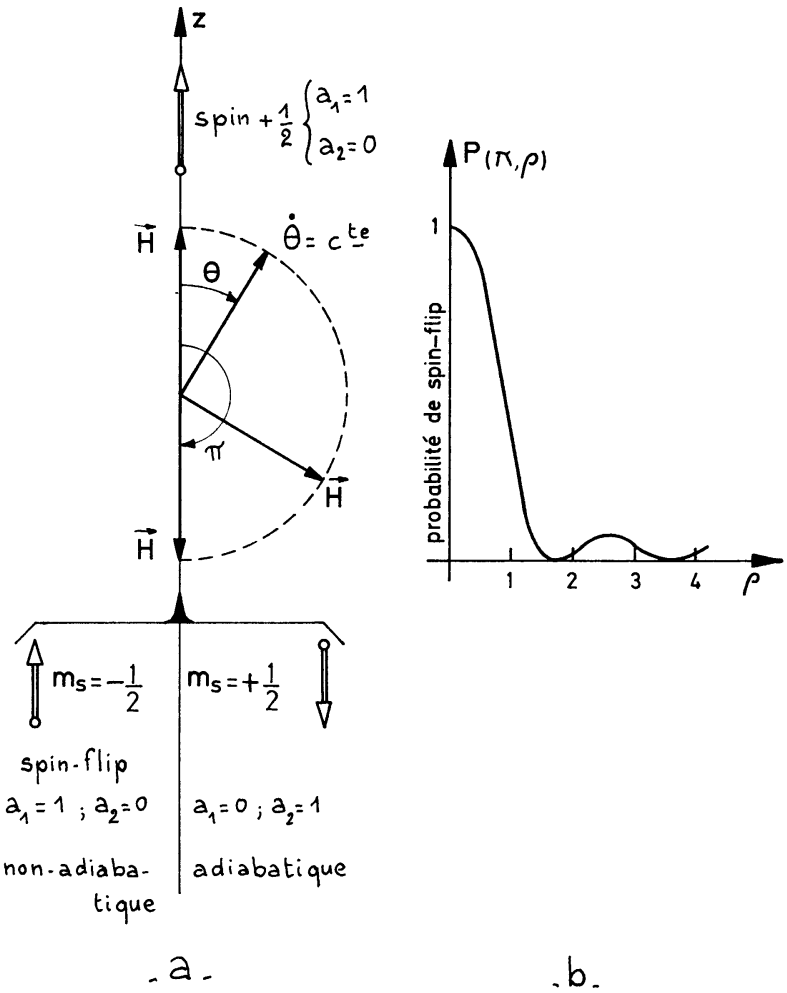

FIG. 17.

riaux ferro- et ferrimagnétiques. Dans ce qui suit nous présentons simplement le mécanisme de base qui définit par les spins une évolution ou adiabatique ou non adiabatique. L'état magnétique d'un $\operatorname{spin} \frac{1}{2}$ est défini au temps $t$ par la fonction

$$
\left|\psi(t)>=a_{1}(t)\right|+\frac{1}{2}>+a_{2}(t) \mid-\frac{1}{2}>
$$

où $a_{1}$ et $a_{2}$ sont les amplitudes de probabilité de la particule dans les états $m_{s}=+\frac{1}{2}$ et $-\frac{1}{2}$ respectivement. L'évolution de la particule dans le champ $H$ est décrite par l'équation de Schrödinger

$$
\mathrm{i} \hbar \delta \psi / \delta t=\boldsymbol{\mu} \mathbf{H} \psi \quad \text { avec } \quad \boldsymbol{\mu}=\gamma \mathbf{S} .
$$

Si au temps $t=0$, le champ $\mathbf{H}$ évolue brusquement de la direction $+o z$ vers la direction $-o z$, on montre que la description de l'évolution de l'état magnétique de la particule est très simple si on adopte un référentiel évoluant avec $\mathbf{H}$. Dans ce référentiel tournant à la vitesse angulaire $\mathrm{d} \theta / \mathrm{d} t$ les deux états propres sont représentés par deux nouvelles amplitudes $b_{1}$ et $b_{2}$ issues de $a_{1}$ et $a_{2}$ par une transformation unitaire. On montre que l'évolution du système magnétique est décrite par l'équation suivante :

$$
\left|\begin{array}{l}
\dot{b}_{1} \\
\dot{b}_{2}
\end{array}\right|=-\frac{1}{2} \mathrm{i} \omega_{0}\left|\begin{array}{rr}
1 & 0 \\
0 & -1
\end{array}\right| \cdot\left|\begin{array}{l}
b_{1} \\
b_{2}
\end{array}\right|-\frac{1}{2} \dot{\theta}\left|\begin{array}{rr}
0 & -1 \\
1 & 0
\end{array}\right| \cdot\left|\begin{array}{l}
b_{1} \\
b_{2}
\end{array}\right|
$$

où $\omega_{0}$ est la fréquence de Larmor de spin associée à $\mathbf{H}$. Au second membre de cette équation, les premier et second termes représentent la matrice des valeurs 
propres et la matrice de couplage entre les états propres. L'importance de ce couplage induit par la variation de $\mathbf{H}$ dépend de la valeur du rapport $\rho=\omega_{0} / \dot{\theta}$. Si $\rho \gg 1$, la rotation de $\mathbf{H}$ est lente comparée à la précession de Larmor. Le terme de couplage de l'équation (17) est négligeable, on obtient une évolution adiabatique du spin, c'est-à-dire que la probabilité d'obtenir un spin-flip d'un état vers l'autre est quasi nulle.

Dans le cas opposé où $\rho \ll 1$ l'équation (17) se réduit en première approximation au terme de couplage :

$$
\mathrm{d} b_{1} / \mathrm{d} t=+\frac{1}{2} \dot{\theta} b_{2} \quad \text { et } \quad \mathrm{d} b_{2} / \mathrm{d} t=-\frac{1}{2} \dot{\theta} b_{1}
$$

ce qui correspond à une rotation rapide de $\mathbf{H}$ par rapport à la vitesse de précession du spin. A partir des équations couplées (18) on montre que le basculement de $\mathbf{H}$ peut induire un spin-flip (Fig. 17) où le spin se trouve antiparallèle à $\mathbf{H}$, c'est-à-dire dans un état excité. Une telle excitation se fait au détriment du champ magnétique d'où une évolution non adiabatique. La probabilité de spin-flip pour $\theta=\pi$ est donnée en fonction de $\rho$ par :

$P(\pi, \rho)=\left|a_{1}(\pi)\right|^{2}=\sin ^{2}\left[(\pi / 2)\left(1+\rho^{2}\right)^{1 / 2}\right] /\left(1+\rho^{2}\right)$.

La figure $17 b$ représente $P(\pi, \rho)$ en fonction du paramètre d'adiabaticité $\rho$. La probabilité de spin-flip est voisine de l'unité pour les cas non adiabatiques, $\rho \ll 1$, qui apparaissent soit lorsque $\omega_{0}$, donc $H$, est faible, soit lorsque la rotation du champ est rapide ( $\theta$ élevé). Par contre pour des champs élevés (fréquence de Larmor élevée) ou tournant lentement, $\rho \gg 1$, et la probabilité de spin-flip est faible. Les oscillations de $P(\pi, \rho)$ sont dues aux «brusques » conditions aux limites adoptées (à $t=0, a_{1}=1$ et $a_{2}=0$ ). Si le champ commence à tourner progressivement les oscillations tendent à disparaître. Dans un matériau ferro- ou ferrimagnétique une évolution non adiabatique s'accompagne par une excitation, non plus localisée sur un spin unique, mais sur l'ensemble des spins. Un basculement rapide du champ est accompagné par l'émission d'un cortège d'ondes de spin qui contribue à accroître le temps de basculement de l'aimantation totale.

2.6 PréCESSION D'UN ENSEMBLE DE SPINS COUPLÉS PAR ÉCHANGE : ONDES DE SPIN ET MAGNONS. - 2.6.1 Magnons ferromagnétiques. - Termes d'échange et de Zeeman : transformations de Holstein-Primakoff. Considérons un matériau ferromagnétique isolant dont les spins sont couplés par interaction d'échange. En présence d'un champ magnétique continu $\mathbf{H}_{0}$ ce système est décrit par l'Hamiltonien de HeisenbergDirac tel que :

$$
\mathscr{H}_{M}=-\sum_{\mathbf{i}>\mathbf{j}} J_{\mathrm{ij}} \mathbf{S}_{\mathbf{i}} \mathbf{S}_{\mathbf{j}}+g \mu_{\mathbf{B}} \mathbf{H}_{0} \sum_{\mathbf{i}} \mathbf{S}_{\mathbf{i}}
$$

où les termes dipolaires de volume et de surface et d'anisotropie magnétocristalline sont négligés. $g, \mu_{\mathbf{B}}$ et $J_{\mathbf{i j}}$ sont le facteur de Landé, le magnéton de Bohr et la constante d'échange. Les modes propres associés aux fluctuations magnétiques de ce système peuvent être déterminés à partir de la théorie de Holstein et Primakoff où les opérateurs de spin $\mathbf{S}_{\mathbf{i}}$ sont exprimés dans une première étape en fonction de leurs composantes circulaires $S_{\mathrm{i}}^{ \pm}=S_{\mathrm{i} x} \pm S_{\mathrm{i} y}$. L'identification des relations caractéristiques de l'oscillateur harmonique quantique avec la propriété bien connue du moment angulaire (éq. (15)) donne les expressions de $S_{\mathrm{i}}^{ \pm}$et $S_{\mathrm{i} z}$ en fonction d'opérateurs de boson $a_{\mathrm{i}}^{+}$ et $a_{\mathrm{t}}$. En négligeant les effets magnétiques non linéaires $\left(a_{\mathrm{i}}^{+} a_{\mathrm{i}}<2 S\right)$ (approximation harmonique) on vérifie que :

$$
\begin{aligned}
& S_{\mathrm{i} z}=S-a_{\mathrm{i}}^{+} a_{\mathrm{i}} ; \quad S_{\mathrm{i}}^{+} \simeq \sqrt{2 S} a_{\mathrm{i}} \\
& S_{\mathrm{i}}^{-} \simeq \sqrt{2 S} a_{\mathrm{i}}^{+} .
\end{aligned}
$$

Il s'ensuit de ces expressions que $a_{\mathrm{i}}^{+}$et $a_{\mathrm{i}}$ apparaissent comme des opérateurs locaux de création et de destruction d'une déviation de spin. S'il est possible d'utiliser ces opérateurs pour décrire les transitions d'un système de spins paramagnétique (Fig. 18a),

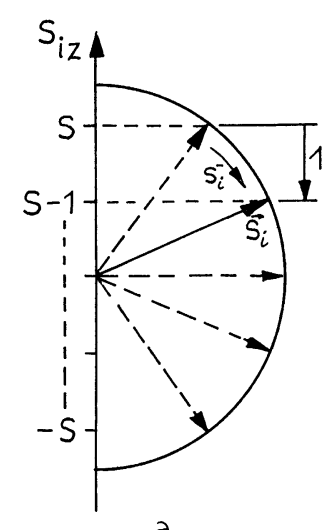

- a .

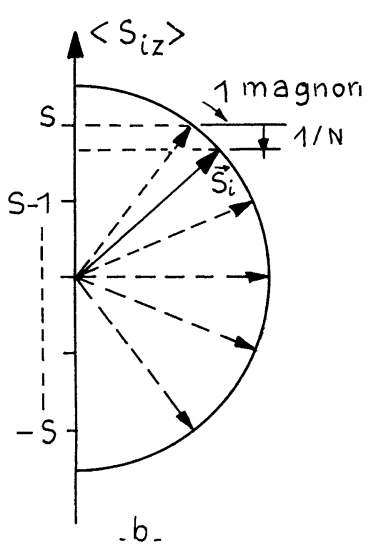

. b.
FIG. 18.

il n'en est plus de même pour des spins couplés par échange. La diagonalisation de 1'Hamiltonien ferromagnétique peut néanmoins être réalisée en développant les opérateurs locaux en série de Fourier :

$$
\begin{aligned}
& S_{\mathrm{i}}^{+} \simeq\left(\frac{2 S}{N}\right)^{1 / 2} \sum_{k} a_{k} \mathrm{e}^{i \mathbf{k} \mathbf{r}_{\mathrm{i}}} ; \\
& S_{\mathrm{i}}^{-} \simeq\left(\frac{2 S}{N}\right)^{1 / 2} \sum_{k} a_{k}^{+} \mathrm{e}^{-i \mathbf{k} \mathbf{r}_{\mathrm{i}}} \\
& S_{\mathrm{i} z}=S=\frac{1}{N} \sum_{1,2} a_{k_{1}}^{+} a_{k_{2}} \exp -i\left(\mathbf{k}_{1}-\mathbf{k}_{2}\right) \mathbf{r}_{\mathrm{i}} .
\end{aligned}
$$

On montre que dans l'approximation harmonique, les excitations magnétiques apparaissent sous la 
forme bien connue d'un ensemble d'oscillateurs harmoniques indépendants décrits par :

$$
\mathscr{H}_{m}=\sum_{k}\left(a_{k}^{+} a_{k}+\frac{1}{2}\right) \hbar \omega_{k}
$$

avec

$$
\hbar \omega_{k}=J S Z\left(1-\gamma_{k}\right)+g \mu_{\mathrm{B}} H_{0}
$$

et

$$
\gamma_{k}=Z^{-1} \sum_{\delta} \mathrm{e}^{i \mathbf{k} \boldsymbol{\delta}}
$$

où $Z$ est le nombre de premiers voisins d'un spin $S_{1}$ donné. Dans le cas des cristaux cubiques on vérifie :

$$
\gamma_{k}=\frac{1}{3}\left(\cos k_{x} a+\cos k_{y} a+\cos k_{z} a\right)
$$

ce qui donne la loi de dispersion des ondes de spin quadratique en $k$ au centre de la première zone de Brillouin. $\hbar \omega_{k}$ étant l'énergie d'excitation magnétique minimale appelée "magnon », $a_{k}^{+}$et $a_{k}$ sont les opérateurs de création et de destruction de magnon. La description semi-classique d'un magnon ferromagnétique est donnée à partir d'un modèle vectoriel dans la figure $18 b$, où on note que la création d'un magnon entraîne en moyenne la diminution de $S_{\mathrm{i} z}$ d'une quantité très faible $1 / N$. Toutes les interactions photon-onde de spin, onde de spin-onde de spin et onde de spin-phonon apparaissent avec échange de un ou de plusieurs quanta. Dans la résonance paramagnétique un photon est absorbé localement par un ion i et $S_{\mathrm{i} z}$ décroît de $S$ à $S-1$. A cause du couplage d'échange le processus d'absorption d'un photon est totalement différent dans un cristal ferromagnétique. La création d'une déviation locale de spin sur un site perturbe énormément l'énergie d'échange au voisinage de ce site. On peut montrer qu'il est énergétiquement plus favorable de répartir cette absorption sur l'ensemble des $N$ spins du cristal qui subissent chacun en moyenne une déviation de $1 / N$. Puisque $N$ est élevé (environ $10^{24}$ dans $1 \mathrm{~cm}^{3}$ ), l'excitation de un magnon produit une perturbation locale de l'énergie d'échange très faible. Ce sont ces considérations physiques qui justifient dans la théorie le remplacement des opérateurs locaux $a_{i}^{+}$par des opérateurs collectifs de magnons $a_{k}^{+}$. Dans une onde de spin tous les spins précessent à la même fréquence $\omega_{k}$ sur des cônes d'angle au sommet identiques comme décrit par le modèle vectoriel, mais en présentant un déphasage spatial de spin à spin d'autant plus grand que la longueur d'onde $\lambda=2 \pi / k$ est petite.

Dans ce qui suit nous utiliserons souvent les opérateurs macroscopiques $\mathbf{M}\left(\mathbf{r}_{\mathbf{i}}\right)$ associés à l'aimantation. Ceux-ci sont liés aux opérateurs de $\operatorname{spin} \mathbf{S}_{\mathrm{i}}$ par :

$$
\mathbf{M}\left(\mathbf{r}_{\mathbf{i}}\right)=g \mu_{\mathrm{B}} \mathbf{S}\left(\mathbf{r}_{\mathrm{i}}\right) \quad \text { avec } \mathbf{S}\left(\mathbf{r}_{\mathbf{i}}\right)=\frac{1}{2} \sum_{\delta} \sigma_{\mathbf{j}} \delta\left(\mathbf{r}_{\mathbf{i}}-\mathbf{r}_{\mathbf{j}}\right)
$$

où $\mathbf{S}\left(\mathbf{r}_{\mathbf{i}}\right)$ et $\sigma_{\mathbf{j}}$ sont respectivement l'opérateur densité de spin au site $\mathbf{r}_{\mathbf{i}}$ et la matrice de Pauli au site $\mathbf{r}_{\mathbf{j}}$. Puisque $\mathbf{M}\left(\mathbf{r}_{\mathrm{i}}\right)=g \mu_{\mathrm{B}} N V^{-1} \mathbf{S}_{\mathrm{i}}$, où $N$ est le nombre de sites magnétiques dans le cristal de volume $V$, les opérateurs aimantations s'expriment en fonction des opérateurs de magnon par les relations suivantes :

$$
\begin{aligned}
& M^{+}(r)=M_{x}+\mathrm{i} M_{y}=\sum_{k}\left(\frac{g \mu_{\mathrm{B}} M_{0}}{2 V}\right)^{1 / 2} a_{k} \mathrm{e}^{i \mathbf{k r}} ; \\
& M^{-}=\left(M^{+}\right)^{*}
\end{aligned}
$$$$
M_{z}(\mathbf{r})=M_{0}-\frac{g \mu_{\mathrm{B}}}{V} \sum_{1,2} a_{k_{1}}^{+} a_{k_{2}} \mathrm{e}^{-i\left(\mathbf{k}_{1}-\mathbf{k}_{2}\right) \mathbf{r}}
$$

avec $M_{0}=g \mu_{\mathrm{B}} N S / V$.

- Termes dipolaires et d'anisotropie magnétocristalline. Transformations de Bogoliubov-Tyablikov. - Au centre de la zone de Brillouin la contribution des termes dipolaires et d'anisotropie magnétocristalline à l'énergie des magnons peut devenir du même ordre de grandeur sinon dépasser la contribution du terme d'échange. Dans ce cas les opérateurs $a_{k}$ définis ci-dessus ne sont plus les vrais opérateurs de magnons et on montre que la précession des spins devient elliptique. On montre que les nouveaux états propres d'excitation magnétique sont obtenus en utilisant les transformations canoniques de Bogoliubov et Tyablilov qui permettent de diagonaliser l'Hamiltonien total du système magnétique. Ces transformations linéaires introduisent de nouveaux opérateurs $c_{k}$ et $c_{k}^{+}$tels que : $a_{k}=u_{k} c_{k}+v_{k}^{*} c_{-k}^{+}$où $u_{k}$ et $v_{k}$ sont des coefficients dépendant des énergies d'échange, dipolaire, d'anisotropie et de Zeeman tel que :

$$
u_{k}=\sqrt{\frac{A_{k}+E_{k}}{2 E_{k}}} \quad v_{k}=-\frac{B_{k}^{*}}{\left|B_{k}\right|} \sqrt{\frac{A_{k}-E_{k}}{2 E_{k}}}
$$

avec :

$$
\begin{aligned}
& A_{k}=D k^{2}+\hbar \omega_{\mathrm{i}}+\frac{\hbar \omega_{m}}{2} \sin ^{2} \theta_{k}+ \\
& +\frac{1}{2}\left(N_{x}^{a_{1}}+N_{x}^{a_{2}}+N_{y}^{a_{1}}+N_{y}^{a_{2}}\right) \hbar \omega_{m} \\
& B_{k}=\frac{\hbar \omega_{m}}{2} \sin ^{2} \theta_{k} \mathrm{e}^{-2 i \varphi_{k}}+ \\
& +\frac{1}{2}\left(N_{x}^{a_{1}}+N_{x}^{a_{2}}-N_{y}^{a_{1}}-N_{y}^{a_{2}}\right) \hbar \omega_{m} \\
& E_{k}=\hbar \omega_{k}=\sqrt{A_{k}^{2}-B_{k} B_{k}^{*}} \\
& \omega_{m}=4 \pi M_{0}, \quad \omega_{\mathrm{i}}=\gamma H_{\mathrm{i}}, \quad H_{\mathrm{i}}=H_{\mathrm{a}}-N_{z} 4 \pi M_{0}
\end{aligned}
$$

$\theta_{k}$ et $\varphi_{k}$ sont respectivement les angles polaire et azimutal du vecteur d'onde $k$ des magnons. $H_{\mathrm{a}}$ est le champ magnétique extérieur appliqué et $N_{z}$ le facteur démagnétisant suivant la direction $O z$ d'application de ce champ. $D$ est une constante d'échange effective. $N_{x}^{a_{1}}$ et $N_{y}^{a_{1}}$ d'une part et $N_{x}^{a_{2}}$ et $N_{y}^{a_{2}}$ d'autre part sont des facteurs d'anisotropie magnétocristalline des premier et second ordres respectivement. Compte tenu de ces transformations les excitations 
magnétiques sont décrites par un nouvel ensemble d'oscillateurs indépendants :

$$
\mathcal{H}_{m}=\sum_{k}\left(c_{k}^{+} c_{k}+\frac{1}{2}\right) \hbar \omega_{k}
$$

L'énergie correcte $\hbar \omega_{k}$ des magnons a une expression complexe qu'on déduit sans difficulté des équations (20):

$$
\begin{gathered}
\hbar \omega_{k}=\left[( D k ^ { 2 } + \hbar \omega _ { i } + 2 \hbar \omega _ { K } ) \left(D k^{2}+\hbar \omega_{i}+\right.\right. \\
\left.\left.+2 \hbar \omega_{K}+\hbar \omega_{m} \sin ^{2} \theta_{k}\right)\right]^{1 / 2} \\
\hbar \omega_{K}=f\left(N_{x}^{a_{1}}, N_{x}^{a_{2}}, N_{y}^{a_{1}}, N_{y}^{a_{2}}\right)
\end{gathered}
$$

(voir éq. (20)).

Cette expression n'est plus valable aux bords de la zone de Brillouin où l'approximation quadratique $D k^{2}$ du terme d'échange doit être remplacée par une expression correcte du type $D\left(1-\cos a_{k}\right)$. Nous vérifions enfin que dans le cas de termes dipolaires et d'anisotropie négligeables $u_{k}=1, v_{k}=0$ et les opérateurs $c_{k}$ se réduisent aux opérateurs simplifiés de magnon $a_{k}$.

La figure 19 décrit le spectre des magnons ferromagnétiques dans la première zone de Brillouin.

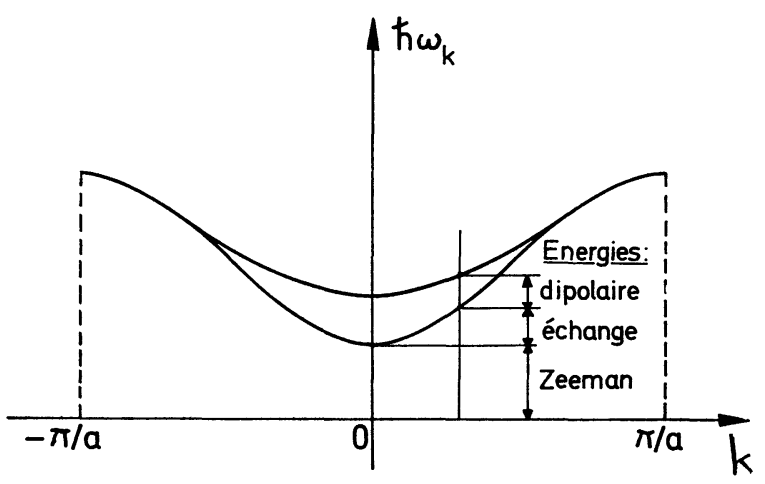

FIG. 19.

2.6.2 Magnons antiferromagnétiques. - En absence de tout champ magnétique extérieur, un cristal antiferromagnétique possède dans chaque maille élémentaire deux sous-réseaux de spins antiparallèles dirigés suivant les directions $+o z$ et $-o z$ par un champ d'anisotropie $H_{\mathrm{A}}$. En présence d'un champ extérieur $\mathbf{H}_{0}$, l'Hamiltonien correspondant s'écrit :

$$
\begin{aligned}
\mathcal{H}_{m}=\sum_{\mathbf{i}, \mathbf{j}} J_{\mathrm{ij}} \mathbf{S}_{\mathbf{i}} \mathbf{S}_{\mathbf{j}} & +g \mu_{\mathrm{B}} H_{\mathrm{A}}\left(\sum_{\mathbf{i}} S_{\mathbf{i}}^{z}-\sum_{\mathbf{j}} S_{\mathbf{j}}^{z}\right) \\
& +g \mu_{\mathbf{B}} H_{0}\left(\sum_{\mathbf{i}} S_{\mathbf{i}}^{z}+\sum_{\mathbf{j}} S_{\mathbf{j}}^{z}\right)
\end{aligned}
$$

(on suppose que $H_{\mathrm{A}}, H_{0}$ et le spin i sont dirigés suivant la direction $+o z$ ). En absence d'excitation magnétique, le spin total de la maille élémentaire est nul $\left(S_{\text {total }}^{z}=S_{\mathrm{i}}^{z}+S_{\mathrm{j}}^{z}=0\right)$. Dans un ferromagnétique l'excitation de un magnon se traduit par une diminution $-1 / N$ de la projection suivant $o z$ pour tous les spins. Dans un antiferromagnétique l'excitation de 1 magnon de vecteur d'onde $k$ entraîne une variation soit de $+1 / N$, soit de $-1 / N$ pour l'ensemble des deux spins antiparallèles de la maille élémentaire. Il existe donc deux modes qu'on notera $\mid \uparrow k>$ et $\mid \downarrow k>$ qui sont dégénérés si $H_{0}=0$. On montre que les modes d'ondes de spin sont obtenus en explicitant l'Hamiltonien ci-dessus en seconde quantification tel que :

$$
\mathscr{H}_{m}=\sum_{k}\left(E_{\downarrow k} C_{\downarrow k}^{+} C_{\downarrow k}+E_{\uparrow k} C_{\uparrow k}^{+} C_{\uparrow k}\right)
$$

avec

$$
\begin{aligned}
& E_{\uparrow k}=\hbar \omega_{k}-g \mu_{\mathrm{B}} H_{\mathrm{O}} \\
& E_{\downarrow k}=\hbar \omega_{k}+g \mu_{\mathrm{B}} H_{0}
\end{aligned}
$$

et

$$
\hbar \omega_{k}=g \mu_{\mathrm{B}} H_{\mathrm{e}}\left[\left(1+H_{\mathrm{A}} / H_{\mathrm{E}}\right)^{2}=\gamma_{k}^{2}\right]^{1 / 2}
$$

où $\gamma_{k}$ est défini plus haut pour les magnons ferromagnétiques et $H_{\mathrm{E}}$ est le champ d'échange. On note qu'il est plus facile de créer un magnon $\mid \uparrow k>$ qu'un magnon $\mid \downarrow k>$. Une description physique simple d'un magnon antiferromagnétique est possible en considérant la projection sur $o z$ des deux spins d'une maille élémentaire :

$$
\left.\begin{array}{l}
<S_{\mathrm{i} z}>=S-\frac{1}{N} \sum_{k} u_{k}^{2} C_{\downarrow k}^{+} C_{\downarrow k}+v_{k}^{2} C_{\uparrow k}^{+} C_{\uparrow k} \\
<S_{\mathrm{j} z}>=-S+\frac{1}{N} \sum_{k} v_{k}^{2} C_{\downarrow k}^{+} C_{\downarrow k}+u_{k}^{2} C_{\uparrow k}^{+} C_{\uparrow k}
\end{array}\right\}
$$

où $u_{k}$ et $v_{k}$ sont des coefficients dépendants de $H_{\mathrm{E}}$, $H_{\mathrm{A}}$ et $k$. $\left(u_{k}\right.$ et $v_{k}$ sont des coefficients différents de ceux qui ont été introduits plus haut pour déterminer les énergies des magnons ferromagnétiques.) Les variations du spin total de la maille élémentaire en fonction des nombres $n_{\downarrow k}$ et $n_{\uparrow k}$ de magnons $\mid \downarrow k>$ et $\mid \uparrow k>$ créés sont déduites de (21) tel que :

$$
\begin{aligned}
<S_{\text {total }}^{z}> & =<S_{\mathrm{ij}}>+<S_{\mathrm{jz}}> \\
& =\frac{1}{N} \sum_{k} n_{\uparrow k}-\frac{1}{N} \sum_{k} n_{\downarrow k} .
\end{aligned}
$$

Les équations (21) montrent que l'état magnétique d'un spin « up » ou "down » dépend à la fois de l'excitation des modes $\mid \uparrow k>$ et $|\downarrow k\rangle$, de leurs populations $C_{\uparrow}^{+} C_{\uparrow}$ et $C_{\downarrow}^{+} C_{\downarrow}$ et des valeurs respectives des coefficients $u_{k}$ et $v_{k}$. On montre que $u_{k}^{2}>v_{k}^{2}$ et que $v_{k} \rightarrow 0$ lorsque $k$ est proche de la limite de la première zone de Brillouin. Dans ce cas $\left(v_{k} \simeq 0\right)$ les équations (21) indiquent que les magnons $\mid \downarrow k>$ et $\mid \uparrow k>$ se propagent uniquement sur les sites $\mathbf{i}$ et $\mathbf{j}$ respectivement. La figure 20 décrit dans un modèle vectoriel l'état de spins $\mathbf{S}_{\mathbf{i}}$ et $\mathbf{S}_{\mathbf{j}}$ en présence d'un magnon $\mid \downarrow k>$ pour $k$ quelconque. On note que la déviation du spin i vers le bas est plus grande $\left(u_{k}^{2} / N\right)$ que la déviation du spin $\mathrm{j}$ vers le haut $\left(v_{k}^{2} / N\right)$. On décrit un magnon $\mid \uparrow k>$ en échangeant $u_{k}$ et $v_{k}$ sur la figure 20. 


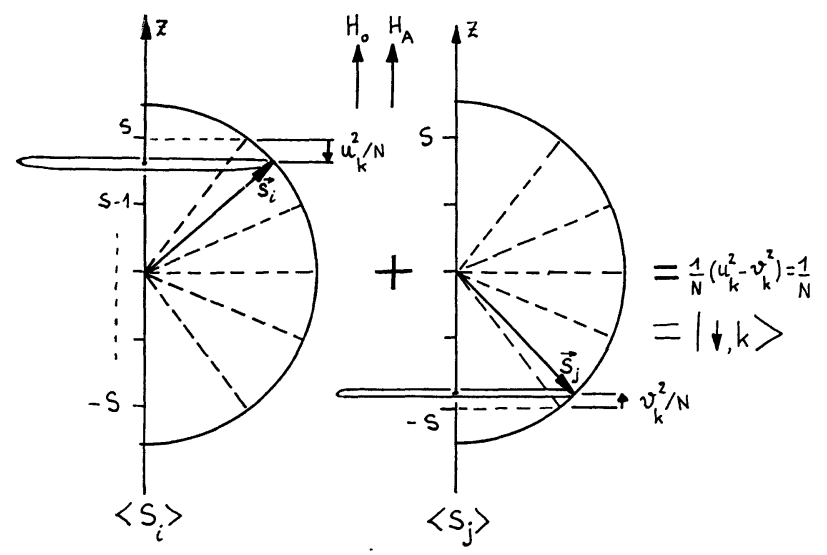

FIG. 20.

2.7 EXCITATION DES ONDES DE SPIN PAR POMPAGES PARALlèle ET PERPENDICUlaire. - L'excitation des ondes de spin par pompage hyperfréquence est définie par le couplage dipolaire magnétique $\mathfrak{H}=-\mathbf{h M}$ qui comprend un terme transverse $\mathfrak{H}_{\perp}$ et un terme longitudinal $\mathscr{H}_{\|}$tel que :

$\mathscr{H}_{\perp}=-\frac{1}{2}\left(M^{+} h^{-}+M^{-} h^{+}\right)$et $\mathscr{H}_{\|}=-M_{z} h_{z}$ associés respectivement aux pompages perpendiculaire et parallèle. Ces expressions peuvent être développées en seconde quantification en explicitant $M^{ \pm}$et $M_{z}$ en opérateurs de magnon et $h$ en opérateurs de photon

$$
\begin{gathered}
\mathbf{h}_{(\mathbf{r}, t)}=\sum_{q} \mathbf{h}^{-}(r) \mathrm{e}^{i \omega q t}+\text { h.c. } \\
h^{-}(\mathbf{r})=-i\left(\frac{2 \pi \hbar \omega_{q}}{\bar{n}^{2} V}\right)^{1 / 2}\left(\mathbf{s} \times \mathbf{e}_{q}^{*}\right) b_{q}^{+} \mathrm{e}^{-i q r}
\end{gathered}
$$

où $\mathbf{e}_{q}=\left(\mathbf{e}_{x}+\mathrm{e}_{y} \mathrm{e}^{i \delta}\right)$ est le vecteur de polarisation complexe du photon $\hbar \omega_{q}$ du vecteur de propagation $\mathbf{q}$. $\bar{n}$ est l'indice de réfraction et $\mathbf{s}=\mathbf{q} /|q|$.

Les énergies dipolaire et d'anisotropie magnétocristalline peuvent contraindre l'évolution des spins à une précession non plus circulaire mais elliptique ce qui induit une composante $M_{2}$ longitudinale de l'aimantation oscillant dans le temps (Fig. 21). Cette composante peut se coupler à une excitation microonde, c'est le pompage "parallèle ». Deux types de transitions apparaissent. Le premier décrit par l'opérateur $c_{k_{1}}^{+} c_{k_{2}} b_{q}$ correspond à l'absorption d'un photon $q$ par un magnon $k_{2}$. La probabilité de transition de ce processus de type Raman peut être considérée comme négligeable en pompage parallèle. Dans le second processus décrit par l'opérateur $c_{k_{1}}^{+} c_{k_{2}}^{+} b_{q}$, un photon est absorbé pour créer deux magnons. Comme généralement $q \simeq 1 \mathrm{~cm}^{-1}$ et $k \gtrsim 10^{4} \mathrm{~cm}^{-1}$, $k_{1} \simeq k_{2}$ et les deux magnons émis ont des vecteurs de propagation égaux mais opposés. Cette interaction est décrite par l'Hamiltonien :

$$
\mathfrak{H}_{\|}=\sum_{k} \Phi_{k} c_{k}^{+} c_{-k}^{+} b_{q}+\text { herm. conj. }
$$

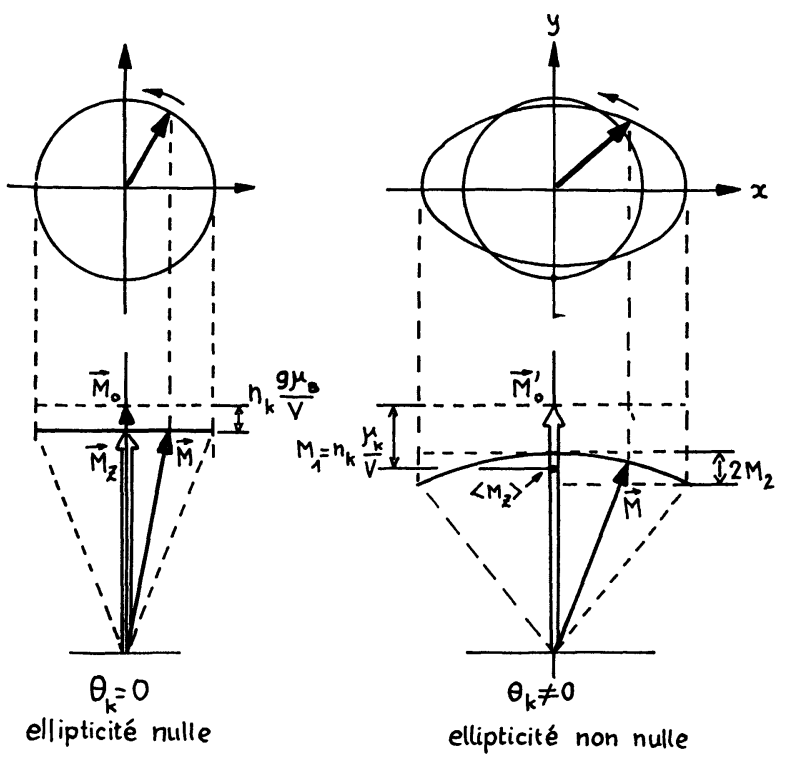

FIG. 21.

où $\Phi_{k}$ dépend de l'énergie dipolaire magnétique des magnons. Le pompage parallèle devient instable à haut niveau d'excitation lorsque le taux de création des magnons est supérieure à leur taux de relaxation. La transition non linéaire à 3 quanta devient alors paramétrique avec le nombre seuil critique de photons donné par: $n_{q}^{\mathrm{c}}=\left(\hbar \eta_{k} / 4\left|\Phi_{k}\right|\right)^{2}$ où $\eta_{k}$ est le taux de relaxation de l'onde de spin $k$. Le pompage parallèle excite des ondes de spin cohérentes dont le vecteur d'onde peut être défini par simple changement du champ magnétique continu. Le taux de relaxation $\eta_{k}$ peut être facilement étudié en fonction de différents paramètres $\left(\omega_{k}, k, T\right.$...) à partir des mesures du seuil critique. Par cette technique il a été possible de vérifier la théorie de la relaxation à 2 , à 3 et à 4 magnons. Le pompage parallèle étant un processus non linéaire la susceptibilité longitudinale correspondante est bien plus faible que la susceptibilité en pompage perpendiculaire $\left(\chi_{1}^{\prime \prime}<0,3\right.$ dans YIG).

Le pompage perpendiculaire est décrit dans des cristaux ferro- ou ferrimagnétique par l'Hamiltonien suivant :

$\mathscr{H}_{\perp}=A\left(b_{q} c_{0}^{+}-b_{q}^{+} c_{0}\right)$ avec $A=-\mathrm{i} \hbar\left(\omega_{m} \omega / 4\right)^{1 / 2}$

qui montre que ce pompage excite uniquement le mode uniforme $(k=0)$. A bas niveau d'excitation, la réponse du système est décrite par le tenseur de susceptibilité magnétique $\chi^{\prime \prime}$ de Polder. $\chi_{\perp}^{\prime \prime}$ est élevé dans les échantillons de faible taux de relaxation $\left(\chi_{\perp}^{\prime \prime} \simeq 700\right.$ dans YIG, mais $\chi_{\perp}^{\prime \prime}=1$ avec $\Delta H=500 \mathrm{Oe}$ ). Audessus d'un certain seuil critique, $\chi_{\perp}^{\prime \prime}$ décroît à cause de l'évolution anharmonique du système qui induit des transitions paramétriques à 3 et 4 magnons du type $c_{0} \quad c_{k}^{+} c_{-k}^{+}$(processus du $1^{\mathrm{er}}$ ordre de Suhl) et 
$c_{0} \quad c_{0} c_{k}^{+} c_{-k}^{+}$(processus du second ordre de Suhl). On peut montrer enfin qu'avec des échantillons de très faible taux de relaxation intrinsèque, une partie importante de l'énergie magnétique est réémise sous forme de transition radiative magnon $\rightarrow$ photon.

2.8 RELAXATION FERRO- ET FERRIMAGNÉTIQUE : INTERACTIONS MAGNONS-MAGNONS ET MAGNONS-PHONONS. Toutes les interactions magnon-magnon sont induites comme l'a montré A. I. Akhiezer [1] par les deux énergies magnétiques de base : l'énergie d'échange $E_{\text {éch }}$ et l'énergie dipolaire $E_{\text {dip }}$ au cristal :

$E_{\text {ech }}=-\sum_{\mathrm{i} \neq \mathrm{j}} J\left(R_{\mathrm{ij}}\right) \mathbf{S}_{\mathrm{i}} \mathbf{S}_{\mathrm{j}}$

$\left.E_{\text {dip }}=\frac{1}{2} \sum_{\mathrm{i} \neq \mathbf{j}} \frac{4 \mu_{\mathrm{B}}^{2}}{R_{\mathrm{ij}}^{5}}\left[R_{\mathrm{ij}}^{2} \mathbf{S}_{\mathrm{i}} \mathbf{S}_{\mathrm{j}}-3\left(\mathbf{R}_{\mathrm{ij}} \mathbf{S}_{\mathrm{i}}\right)\left(\mathbf{R}_{\mathrm{ij}} \mathbf{S}_{\mathrm{j}}\right)\right]\right\}$

Ces énergies dépendent de la distance $R_{\mathrm{ij}}$ entre deux sites magnétiques. Toute variation $\Delta R_{\mathrm{ij}}$ de distance interatomique associée aux phonons entraîne une variation de ces énergies magnétiques [1]. Cette variation est à l'origine des transitions magnonphonon :

$$
\begin{aligned}
& \Delta E_{\mathrm{ech}}=-\sum_{\mathrm{i}, \mathrm{j}} \frac{\delta J}{\delta R_{\mathrm{ij}}} \Delta R_{\mathrm{ij}} \times \\
& \times\left[S_{\mathrm{i}}^{z} S_{\mathrm{j}}^{z}+\frac{1}{2}\left(S_{\mathrm{i}}^{+} S_{\mathrm{j}}^{-}+S_{\mathrm{i}}^{-} S_{\mathrm{j}}^{+}\right)\right] \\
& \Delta E_{\mathrm{dip}}=\sum_{\mathrm{i}, \mathrm{j}} \frac{\mu_{\mathrm{B}}^{2}}{R_{\mathrm{ij}}^{5}}\left\{\left[30 \frac{\left(\mathbf{R}_{\mathrm{ij}} \mathbf{S}_{\mathrm{i}}\right)\left(\mathbf{R}_{\mathrm{ij}} \mathbf{S}_{\mathrm{j}}\right) \mathbf{R}_{\mathrm{ij}}}{R_{\mathrm{ij}}^{2}}-\right.\right. \\
& \left.\left.-12\left(\mathbf{S}_{\mathrm{i}} \mathbf{R}_{\mathrm{ij}}\right) \mathbf{S}_{\mathrm{j}}\right] \Delta \mathbf{R}_{\mathrm{ij}}-6 \mathbf{R}_{\mathrm{ij}} \Delta \mathbf{R}_{\mathrm{ij}} \mathbf{S}_{\mathrm{i}} \mathbf{S}_{\mathbf{j}}\right\}
\end{aligned}
$$

Ces deux couplages magnétoélastiques sont décrits par un modèle d'interaction entre paire d'ions. L'interaction spin-orbite décrit également, mais dans un modèle à un ion, certaines transitions spin-phonon : $E_{\text {so }}=\lambda \mathbf{L S}$. En effet toute modulation du champ cristallin par la présence des phonons modifie l'état du spin par l'intermédiaire d'un mouvement orbital perturbé et du couplage spin-orbite.

La relaxation ferrimagnétique peut être analysée à partir des processus 1 à 11 des figures 22 et 23 qui décrivent l'ensemble des mécanismes de transfert énergétique pouvant apparaître entre une excitation électromagnétique microonde et les différents états magnétiques d'un cristal ferrimagnétique. Après excitation (processus 1) par interaction dipolaire magnétique, l'amplitude du mode de magnon $k_{1}$ décroît soit par désexcitation radiative (processus dipolaire 2) soit par désexcitation non radiative (processus 3 à 11) qui sont à l'origine de la relaxation magnétique telle qu'elle nous intéresse en pompage perpendiculaire ou parallèle. L'origine microscopique des processus 3 à 11 est définie par les interactions d'échange, dipolaire et spin-orbite et par leurs variations avec les déplacements élastiques. La relaxation magnétique décrit le transfert d'énergie entre un mode

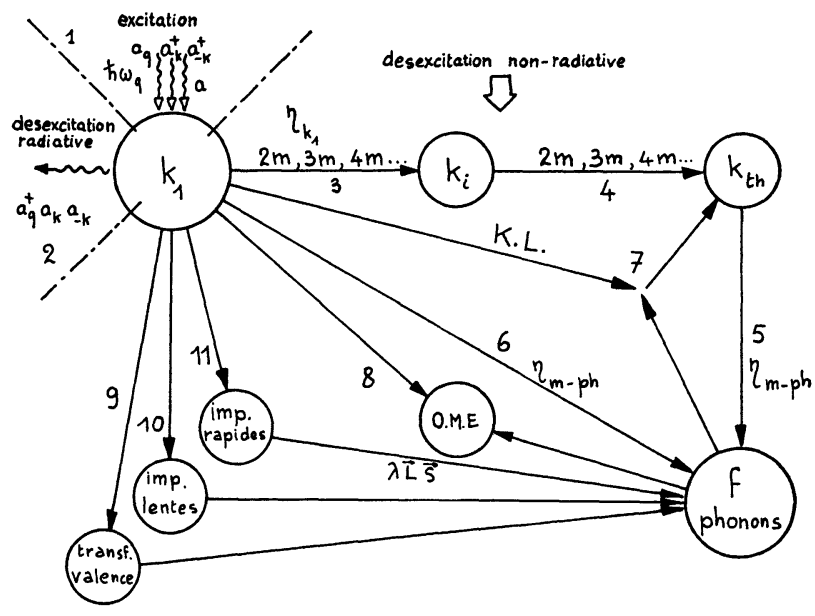

FIG. 22.

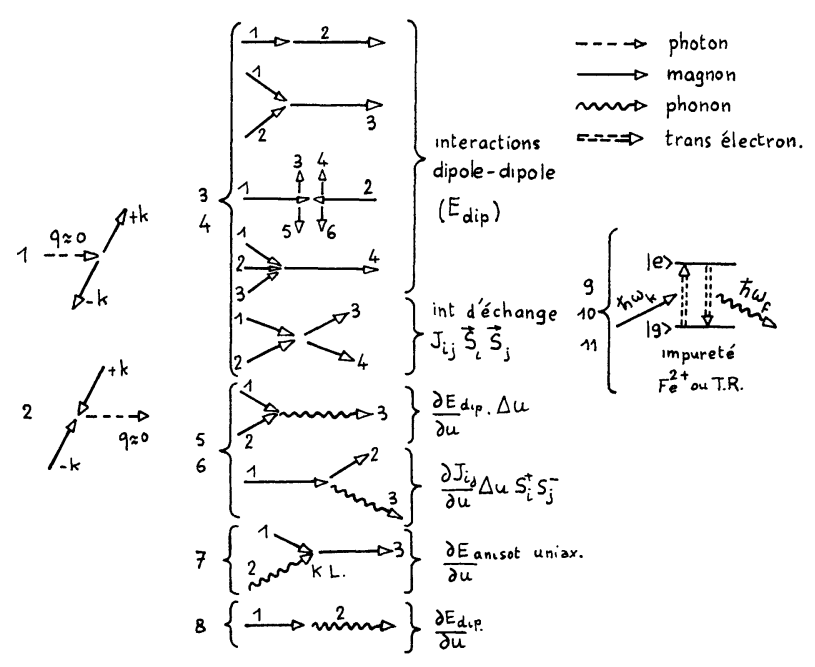

FIG. 23.

initial de magnon $k_{1}$ et le système de phonon $\mathrm{f}$. On peut distinguer trois types de transferts :

- les transferts « magnétiques » (processus 3 et 4),

- les transferts « magnétoélastiques » (processus 5,

6, 7 et 8),

- les transferts par « impuretés » (processus 9, 10 et 11).

2.8.1 Transferts magnétiques. - En appliquant aux énergies d'échange et dipolaire du cristal (éq. (22)), les différentes transformations de Holstein et Primakoff $\left(\mathbf{S}_{\mathbf{i}} \rightarrow S_{\mathrm{i}}^{ \pm} \rightarrow a_{\mathrm{i}}^{+} \rightarrow a_{k}^{+}\right)$puis de Bogoliubov et Tyablikov $\left(a_{k}^{+} \rightarrow C_{k}^{+}\right)$, on fait apparaître différents termes d'interaction décrits par des hamiltoniens du type :

$$
\begin{gathered}
C_{12} b_{1} b_{2}^{+}, C_{123} b_{1} b_{2} b_{3}^{+}, C_{1234} b_{1} b_{2} b_{3} b_{4}^{+}, \\
C_{1234}^{\prime} b_{1} b_{2} b_{3}^{+} b_{4}^{+}, \text {etc... }
\end{gathered}
$$

où $C_{12}, C_{123}$ et $C_{1234}$ représentent des coefficients de couplage à 2,3 et 4 magnons. Une description physique de l'interaction entre magnons est aisée 
à comprendre. Considérons par exemple le processus de fusion à 3 magnons $C_{123} b_{1} b_{2} b_{3}^{+}$induit par couplage dipolaire magnétique. Une interaction dipolaire entre magnons apparaît parce que le dipôle magnétique $\mu_{1}$ du magnon $k_{1}$ produit un champ démagnétisant $\mathbf{h}_{\mathrm{d}_{1}}$. Dans un tel champ, le dipôle magnétique $\boldsymbol{\mu}_{2}$ d'un magnon $k_{2}$ possède une énergie $-\boldsymbol{\mu}_{2} \mathbf{h}_{\mathrm{d}_{1}}$ qui s'explicite, en seconde quantification. par les termes (24) écrits ci-dessus.

2.8.2 Transferts magnétoélastiques. - L'introduction du formalisme de seconde quantification dans les termes magnétoélastiques (23) fait apparaître 4 transitions magnon-phonon principales décrites par des hamiltoniens du type :

$$
\begin{aligned}
& \Delta E_{\mathrm{dip}} \rightarrow\left\{\begin{array}{l}
D_{\mathrm{me}} b_{1} d_{1}^{+}+\text {h. c. } \\
D_{2 \mathrm{~m}-1 \mathrm{ph}} b_{1} b_{2} d_{3}^{+}+\text {h. c. } \\
D_{\mathrm{K} . \mathrm{L} .} b_{1} d_{2} b_{2}^{+}+\text {h. c. } \\
D_{\text {Cérenkov }} b_{1} d_{2}^{+} b_{3}^{+}+\text {h. c. }
\end{array}\right\} \\
& \Delta E_{\text {ech }} \rightarrow
\end{aligned}
$$

( $b=$ opérateur de magnon, $d=$ opérateur de phonon).

La transition $D_{\mathrm{me}} b_{1} d_{1}^{+}+$h. c. représente les ondes magnéto élastiques apparaissant au croisement des spectres des phonons et des magnons. La transition $D_{2 \mathrm{~m}-1 \mathrm{ph}} b_{1} b_{2} d_{3}^{+}$entraîne la création d'un phonon à partir de la destruction de deux magnons. $D_{\mathrm{K} . \mathrm{L} .} b_{1} d_{2} b_{3}^{+}$représente la création d'un magnon à partir de la destruction simultanée d'un magnon de faible énergie et d'un phonon de grande énergie. C'est le processus invoqué par Kasuya et Le Craw [2] pour expliquer le taux de relaxation résiduel des magnons $k \simeq 0$. Le terme $D_{\text {Cérenkov }} b_{1} d_{2}^{+} b_{3}^{+}$représente une interaction magnon-phonon du type Cérenkov. Dans de telles transitions un magnon ne cède une partie de son énergie au système élastique que si la vitesse de groupe du magnon incident est égale ou supérieure à la vitesse du son:

$$
\frac{\delta \omega_{k}}{\delta k} \geqslant \frac{\delta \omega_{\mathrm{f}}}{\delta \mathrm{f}} \rightarrow k>\frac{\hbar v_{\mathrm{son}}}{2 \mathrm{D}} \simeq 4 \times 10^{6} \mathrm{~cm}^{-1} .
$$

C'est le mécanisme qui est le plus efficace pour trans- férer, en fin de relaxation, l'énergie du système des magnons vers celui des phonons [1].

2.8.3 Transferts par impuretés. - Contrairement aux transferts magnétiques et magnétoélastiques qui définissent tous (exception faite pour les transitions à 2 magnons) des interactions délocalisées, les relaxations par impuretés correspondent à des processus localisés. L'interaction spin-réseau apparaît par l'intermédiaire des états excités des impuretés ce qui implique un transfert d'énergie par deux transitions réelles de temps de relaxation $T_{m}$ et $T_{R}$. La relaxation magnonphonon globale devient : $T_{k}^{-1}=T_{m}^{-1}+T_{R}^{-1}$. Dans les grenats ferrimagnétiques les impuretés sont principalement constituées par des ions $\mathrm{Fe}^{2+}$ ou des ions de terres rares $\mathrm{Yb}^{3+}, \mathrm{Tb}^{3+}, \mathrm{Sm}^{3+}, \mathrm{Ho}^{3+} \ldots$ Ces impuretés possèdent une interaction spin-orbite importante qui produit un couplage impureté-phonon très élevé. Par la première transition réelle $T_{m}$, la destruction d'un magnon uniforme $\hbar \omega_{u}$, ou non uniforme $\hbar \omega_{k}$, provoque l'excitation d'une impureté. On distingue généralement trois types de transition :

- transitions par transfert de valence

$$
\mathrm{Fe}^{2+}-3 \mathrm{~d}^{6}\left({ }^{5} \mathrm{D}\right) \rightarrow \mathrm{Fe}^{3+}-3 \mathrm{~d}^{5}\left({ }^{6} \mathrm{~S}\right),
$$

- transitions par échange anisotrope $\mathrm{Fe}^{3+}$-Terre rare

$$
\mathcal{H}_{\mathrm{AB}}=\sum_{\mathrm{i}, \mathrm{j}=1}^{3} \hbar \omega_{\mathrm{AB}}^{\mathrm{ij}} S_{\mathrm{A}}^{\mathrm{i}} S_{\mathrm{B}}^{\mathrm{j}},
$$

- transitions par échange isotrope $\mathrm{Fe}^{3+}-$ Terre rare

$$
\begin{aligned}
& \mathscr{H}_{\mathrm{AB}}=-\hbar \omega_{\mathrm{AB}} \mathbf{S}_{\mathrm{A}} \mathbf{S}_{\mathrm{B}}= \\
& \quad=-\hbar \omega_{\mathrm{AB}}\left[S_{\mathrm{A}}^{z} S_{\mathrm{B}}^{z}+\frac{1}{2}\left(S_{\mathrm{A}}^{+} S_{\mathrm{B}}^{-}+S_{\mathrm{A}}^{-} S_{\mathrm{B}}^{+}\right)\right]
\end{aligned}
$$

où $\mathrm{A}$ et $\mathrm{B}$ sont respectivement un ion $\mathrm{Fe}^{3+}$ et un ion de terre rare. Dans ce dernier cas les impuretés sont supposées avoir une "relaxation rapide». Par comparaison avec ce cas, les transitions induites par l'échange anisotrope sont associées à des impuretés ayant une « relaxation lente $»$.

Nous avons ainsi le schéma général :

$$
\frac{1}{T_{k}}=\frac{1}{T_{m}}+\frac{1}{T_{R}}
$$

magnons

phonons

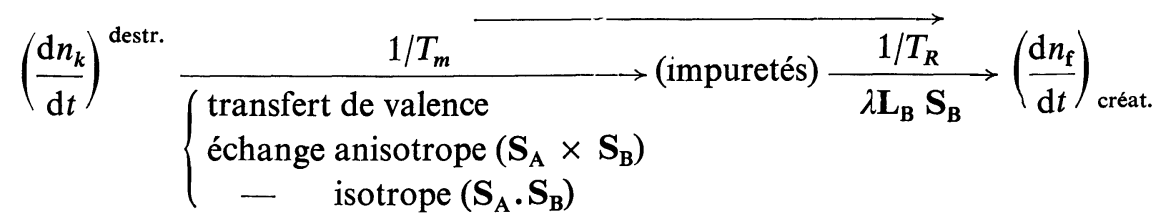

Les trois mécanismes par impuretés possèdent une forte dépendance en température et on vérifie expérimentalement que la relaxation des magnons croît brutalement pour $T<100 \mathrm{~K}$ environ, Nous allons décrire plus en détail les trois mécanismes associés aux impuretés. 
2.8.4 Transfert de valence: impuretés du type $\mathrm{Fe}^{2+}$. - Les ions $\mathrm{Fe}^{3+}$ étant des états $\mathrm{S}$ (couplage spinorbite et anisotropie magnétocristalline négligeables), les niveaux d'énergie des multiplets de base ne sont pas perturbés ou déplacés au cours de la précession de l'aimantation. Il n'en est pas de même pour les multiplets de base des ions $\mathrm{Fe}^{2+}$ qui par le couplage spin-orbite subissent une forte influence du champ cristallin qui varie périodiquement avec l'angle de précession $\varphi=\omega_{k} t$. La variation périodique de l'énergie des ions $\mathrm{Fe}^{2+}$ avec l'angle de précession est schématisée sur la figure 24 où $\mathbf{I}$ et $\mathbf{J}$ représentent deux sites voisins entre lesquels apparaît un transfert d'électron. Comme indiqué sur cette figure, à un instant donné $t_{1}$, l'électron supplémentaire tend à sauter du site I d'énergie plus élevée vers le site $J$ d'énergie plus basse en émettant, par le couplage spin-orbite, un phonon. Ce transfert d'énergie est fait aux dépens des magnons. Une demi-période après $t_{1}$ une seconde transition peut apparaittre mais cette fois du site $J$ vers le site I.

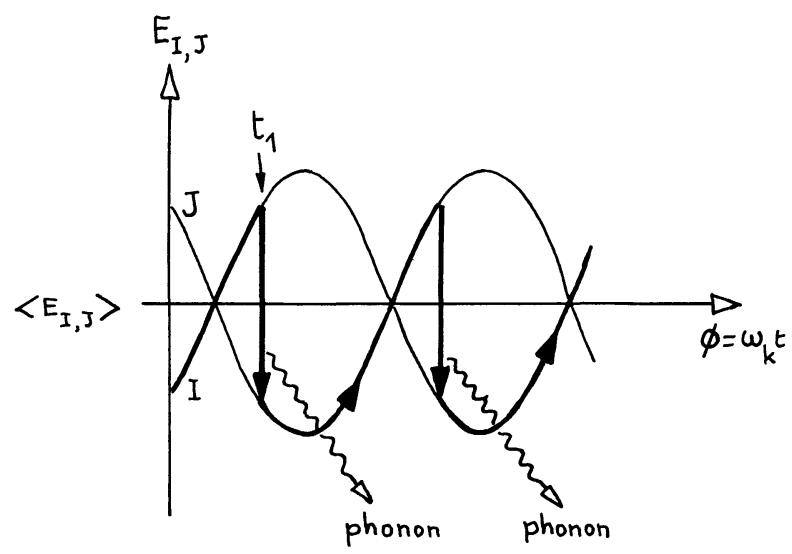

FIG. 24.

\subsubsection{Echange anisotrope et impuretés de relaxation} lente. - L'ion $\mathrm{Yb}^{3+}$ dans le YIG possède un doublet de base dont la dégénérescence est levée par l'interaction d'échange anisotrope qui apparaît entre les ions ferrimagnétiques $\mathrm{Fe}^{3+}$ et l'ion $\mathrm{Yb}^{3+}$. Puisque cet échange est anisotrope les niveaux du doublet $E_{\mathrm{I}}$ et $E_{\mathrm{J}}$ sont fonction de l'orientation de l'aimantation des ions $\mathrm{Fe}^{3+}$ et doivent ainsi varier au cours de la précession ferrimagnétique. Un tel résultat, schématisé sur la figure 25, est décrit par des termes tels que $S_{\mathrm{A}}^{x} S_{\mathrm{B}}^{z}$ qui représentent l'action d'un champ $S_{\mathrm{A}}^{x}$ oscillant à la fréquence des magnons et agissant, tel un hamiltonien de Zeeman, sur le spin $\mathbf{S}_{\mathrm{b}}$ suivant la direction $O z$ :

$$
\mathscr{H}_{\mathbf{A B}}=-\mathbf{H}_{\mathbf{A}} \mathbf{S}_{\mathbf{B}} \text {. }
$$

Ainsi les niveaux $E_{\mathrm{I}}$ et $E_{\mathrm{J}}$ de l'impureté sont modulés à la fréquence $\omega_{k}$ par ce champ r. f. A ce stade le processus de relaxation de l'impureté est analogue à celui du transfert de valence décrit précédemment. En effet les taux d'occupation relatifs des niveaux I et $\mathbf{J}$

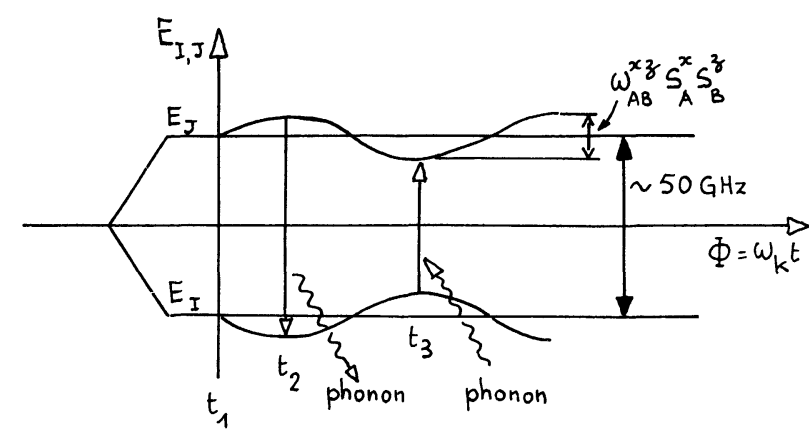

FIG. 25.

sont fonction de $\varphi$ par les facteurs de Boltzmann $\exp -\left(E_{\mathrm{I}, \mathrm{J}} / k_{\mathrm{B}} T\right)$. Puisque la séparation énergétique $\left(E_{\mathrm{J}}-E_{\mathrm{I}}\right)$ croît entre $t_{1}$ et $t_{2}$, un nouvel état d'équilibre thermodynamique ne peut être atteint qu'après apparition de transitions $\mathbf{J} \rightarrow$ I accompagnées d'émission de phonon $\hbar \omega_{\mathrm{f}}(2)=E_{\mathrm{J}}\left(t_{2}\right)-E_{\mathrm{I}}\left(t_{2}\right)$. Par contre en $t_{3}$ les processus inverses accompagnés d'absorption de phonons $\hbar \omega_{\mathrm{f}}(3)=E_{\mathrm{J}}\left(t_{3}\right)-E_{\mathrm{I}}\left(t_{3}\right)$ sont nécessaires pour définir une nouvelle distribution de Boltzmann. La perte nette d'énergie par cycle de précession détermine le taux de relaxation $T_{k}$.

Pour $T \rightarrow 0 \mathrm{~K}$ seul l'état I est occupé

$$
\left(E_{\mathrm{J}}-E_{\mathrm{I}} \sim 25 \mathrm{~K}\right)
$$

donc des transitions $\mathrm{I} \rightarrow \mathbf{J}$ puis $\mathbf{J} \rightarrow \mathbf{I}$ sont inexistantes et $1 / T_{R} \rightarrow 0$. On obtient un résultat identique pour des températures très élevées où les taux d'occupation des niveaux $I$ et $J$ devenant égaux, aucun transfert net d'énergie vers les phonons n'apparaît plus. Il existe une température intermédiaire pour laquelle la condition $\omega_{k} \cdot T_{R} \simeq 1$ est réalisée et qui entraîne un maximum de la relaxation dite "lente ".

2.8.6 Echange isotrope et impuretés de relaxation rapide. - Le troisième processus de relaxation par impureté nécessite, contrairement au cas précédent, une interaction d'échange isotrope $S_{A} S_{B}$ entre l'impureté de terre rare $\mathrm{B}$ et les ions $\mathrm{A}\left(\mathrm{Fe}^{3+}\right)$. Dans ce mécanisme, les échanges énergétiques $\mathrm{A} \rightleftarrows \mathrm{B}$ sont décrits par les termes $S_{\mathrm{A}}^{+} S_{\mathrm{B}}^{-}$et $S_{\mathrm{A}}^{-} S_{\mathrm{B}}^{+}$. La relaxation apparaît ainsi par l'intermédiaire d'une première transition où un magnon $\hbar \omega_{k}$ est absorbé pour transférer l'ion de terre rare d'un état de base $|\mathrm{g}\rangle$ vers un état excité $|\mathrm{n}\rangle$. On montre que la séparation énergétique $E_{\mathrm{n}}-E_{\mathrm{g}}$ de l'impureté est induite par l'échange isotrope tel que $E_{\mathrm{n}}-E_{\mathrm{g}}=\hbar \omega_{\mathrm{ng}} \simeq \hbar \omega_{\mathrm{AB}}$ $(\sim 5 \mathrm{~K}$ ou $50 \mathrm{GHz}$ ). Comme les magnons ont une énergie de l'ordre de $0,5 \mathrm{~K}$ (ou 1 à $10 \mathrm{GHz}$ ), les transitions du type $b_{k} S_{\mathrm{B}}$ ne peuvent être, en règle générale, que virtuelles (Fig. 26a) sauf si la largeur de raie naturelle $\Delta E$ du niveau $|\mathrm{n}\rangle$ ou $|\mathrm{g}\rangle$ est équivalente à la séparation $E_{\mathrm{n}}-E_{\mathrm{g}}:$ (Fig. 26b) :

$$
\hbar \omega_{\mathrm{ng}} \simeq \hbar \omega_{\mathrm{AB}} \simeq \Delta E(\sim 50 \mathrm{GHz}) .
$$

La relation d'incertitude montre que le temps de 
relaxation $T_{R}$ du niveau excité $|\mathrm{n}\rangle$ est inversement proportionnel à $\Delta E\left(T_{R} \simeq \hbar / \Delta E\right)$, ce qui entraîne : $\omega_{\mathrm{AB}} \cdot T_{R} \simeq 1$ ou $T_{R} \simeq 1 / \omega_{\mathrm{AB}} \simeq 2 \times 10^{-12} \mathrm{~s}$.

Ainsi le transfert $\mathrm{A} \rightarrow \mathrm{B}$ devient une transition réelle (Fig. 26b), à la condition que le temps de relaxation de l'impureté soit très rapide pour induire un

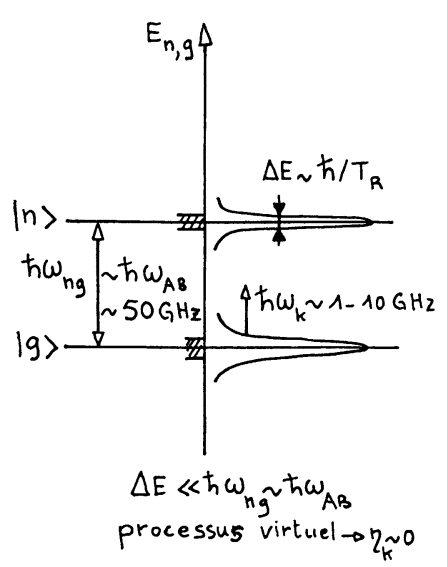

-a.

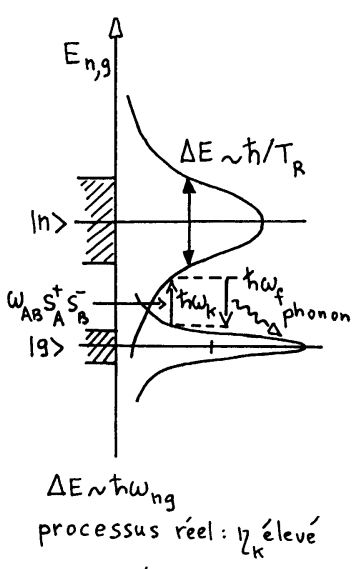

- b.
Fig. 26.

élargissement $\Delta E$ suffisant de ses niveaux. Après excitation, l'impureté de terre rare revient à son état de base en émettant un phonon. Dans le cas limite $\omega_{\mathrm{AB}} T_{R} \ll 1$ ou $\Delta E \gg \hbar \omega_{\mathrm{ng}}$, la relaxation tend vers zéro car seul un nombre restreint d'ions de terre rare possède la séparation $\hbar \omega_{k}$ nécessaire à la transition $\mathrm{A} \rightarrow \mathrm{B}$.

En conclusion, on montre que la relaxation est maximale pour les mécanismes associés aux échanges isotrope et anisotrope lorsque :

Echange isotrope : $\omega_{\mathrm{AB}} \cdot T_{R}=1, T_{R} \simeq 2 \times 10^{-12} \mathrm{~s}:$ relaxation « rapide".

Echange anisotrope $: \omega_{k} \cdot T_{R}=1, T_{R} \simeq 2 \times 10^{-10} \mathrm{~s}:$ relaxation «lente ».

C'est ce qui justifie les appellations d'amortissements par impuretés de relaxation rapide et lente.

3. Mouvement de spin dans la bande optique. Bigyrotropisme et magnéto-optique. - 3.1 LES DIFFÉRENTES INTERACTIONS MAGNÉTO-OPTIQUES. - Les interactions spin-photon sont décrites dans la bande optique par les phénomènes magnéto-optiques. Si le nombre des effets magnéto-optiques connus depuis plus d'un siècle a été relativement restreint, et nous citerons les effets Faraday (1845), Kerr magnétique (1876), Zeeman (1896) et Cotton-Mouton ou Voigt (1906), il n'en reste pas moins vrai qu'ils ont été d'une importance capitale dans l'évolution de la connaissance scientifique et tout particulièrement en ce qui concerne la mise en évidence de la nature électromagnétique de la lumière. Ce n'est que récemment avec l'utilisation des techniques laser, que nous avons assisté à une véritable «explosion" de nouveaux mécanismes d'interaction spin-photon que nous pouvons classer en trois groupes. Dans le premier groupe figurent l'absorption à 2 magnons d'abord observée dans l'infrarouge lointain sur le fluorure antiferromagnétique $\mathrm{FeF}_{2}$ (1964) et l'absorption 2 magnons-1 phonon détectée dans un cristal ferromagnétique de nickel (1959) puis dans le cristal antiferromagnétique $\mathrm{KNiF}_{3}$ (1966). Dans ces processus un photon est absorbé et deux magnons, ou deux magnons plus un phonon, sont créés. Le second groupe correspond aux interactions exciton-magnon ou bandes latérales de magnons (" magnon side-band »). Un photon est alors absorbé ou émis et un magnon plus un exciton sont créés ou détruits. Le premier cas est l'absorption magnon-exciton découverte dans $\mathrm{MnF}_{2}$ (1966). Le second cas est l'émission ou fluorescence exciton-magnon observée aussi dans $\mathrm{FeF}_{2}$ (1967) puis dans $\mathrm{EuO}$ (1971). Le troisième groupe s'adresse aux diffusions Raman de spin d'abord mises en évidence sur des ions $\operatorname{Pr}^{3+}$ substitués dans $\mathrm{LaF}_{3}$ (1963). Dans les cristaux ferro-, ferri- et antiferromagnétiques, les effets Raman de spin deviennent les effets Raman à 1,2 et 4 magnons où la diffusion de 1 photon s'accompagne de la création (processus Stokes) ou de la destruction (processus anti-Stokes) de 1, 2 ou 4 magnons. Les premières expériences ont été réalisées dans $\mathrm{FeF}_{2}$ et $\mathrm{MnF}_{2}$ (diffusion Raman à 1 et 2 magnons) puis dans $\mathrm{NiO}$ et $\mathrm{KNiF}_{3}$ (diffusion à 4 magnons).

Une classification intéressante des interactions spinphoton est obtenue en séparant les processus d'absorption (ou d'émission) des processus dispersifs qui induisent la diffusion de la lumière par le cristal magnétique comme indiqué dans le tableau page suivante :

3.2 DESCRIPTION MACROSCOPIQUE DES EFFETS MAGNÉTO-OPTIQUES : LES DIPÔLES INDUITS. - La description macroscopique, d'abord classique, puis quantique des effets magnéto-optiques ne présente aucune difficulté en utilisant comme point de départ les dipôles électriques $\mathbf{P}_{q}$ et magnétiques $\mathbf{m}_{q}$ induits par le rayonnement de fréquence $\omega_{q}$ dans le cristal magnétique. A cet effet nous allons définir successivement ces deux dipôles.

Le dipôle électrique est obtenu en partant du tenseur de permittivité $\varepsilon_{\mathrm{ij}}$ qui comprend des composantes hermitique $\varepsilon_{\mathrm{ij}}^{\mathrm{H}}$ et antihermitique $\varepsilon_{\mathrm{ij}}^{\mathrm{A}}$ associés respectivement avec les phénomènes de dispersion et d'absorption $\left(\varepsilon_{\mathbf{i j}}=\varepsilon_{\mathbf{i j}}^{\mathbf{H}}+\varepsilon_{\mathbf{i j}}^{\mathbf{A}}\right)$. Nous limitons dans ce qui suit l'étude aux phénomènes dispersifs (on suppose le cristal quasi transparent $\varepsilon_{\mathbf{i j}}^{\mathbf{A}} \simeq 0$ ), aussi $\varepsilon_{\mathrm{ij}}$ se réduit à sa composante hermitique qui est généralement complexe $\left(\varepsilon_{\mathrm{ij}} \simeq \varepsilon_{\mathrm{ij}}^{\mathrm{H}}=\varepsilon_{\mathrm{ij}}^{\prime}+\mathrm{i} \varepsilon_{\mathrm{ij}}^{\prime \prime}\right)$. A partir des propriétés de symétrie du tenseur de permittivité dépendant du spin $\left(\varepsilon_{\mathrm{ij}}(\mathrm{M})=\varepsilon_{\mathrm{ji}}(-\mathrm{M})\right)$, on montre sans difficulté que $\varepsilon^{\prime}$ et $\varepsilon^{\prime \prime}$ représentent respectivement les 


\section{TABLEAU I}

Interactions spin-photon

$$
\text { 1er ordre }
$$

$2^{\mathrm{e}}$ ordre

I. Interactions dipolaires $\left\{\begin{array}{l}\text { magnétique } \mu_{\mathrm{ij}}=\mu_{\mathrm{ij}}^{(0)}+a_{\mathrm{ij} k} M_{k}+a_{\mathrm{ijkl}} M_{k} M_{l} \\ \text { électrique } \varepsilon_{\mathrm{ij}}=\varepsilon_{\mathrm{ij}}^{(0)}+b_{\mathrm{ijk}} M_{k}+b_{\mathrm{ijkl}} M_{k} M_{l}\end{array}\right.$

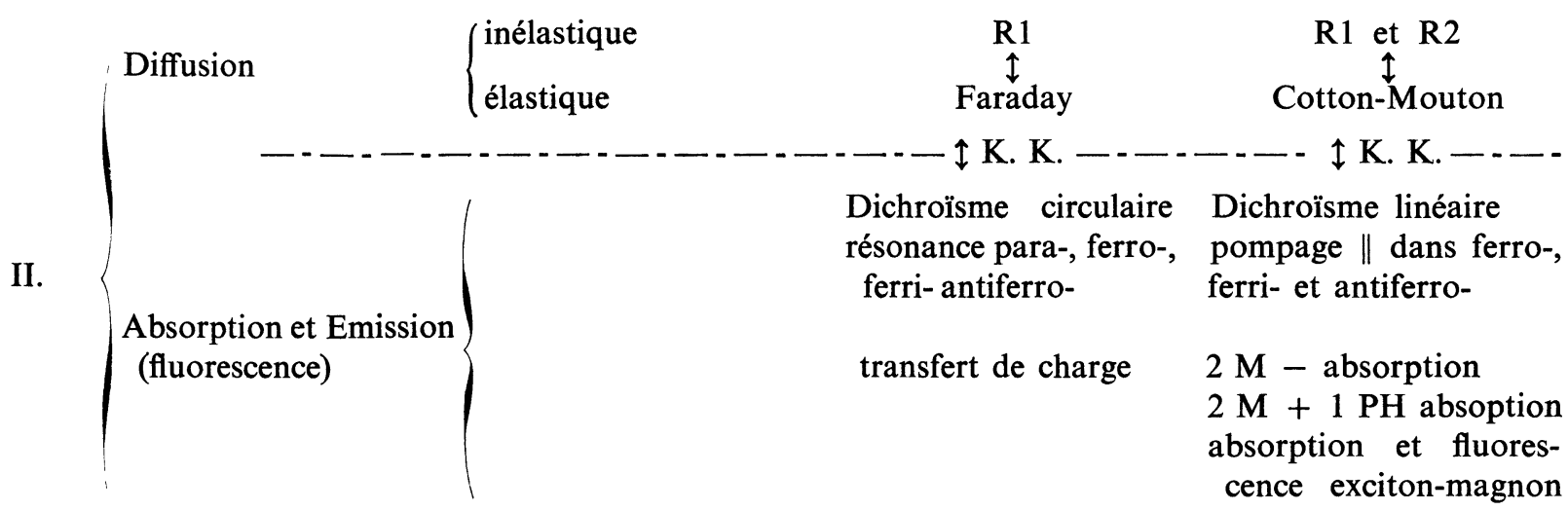

K. K. = relations de Kramers-Kronigs

$2 \mathrm{M}=$ deux magnons $; 1 \mathrm{PH}=$ un phonon.

effets M. O. pairs et impairs. On déduit de ce tenseur les dipôles électriques tel que :

$$
\begin{gathered}
\mathbf{P}=\sum_{q} \mathbf{P}_{q}^{+} \mathrm{e}^{-i \omega_{q} t}+\mathbf{P}_{q}^{-} \mathrm{e}^{i \omega_{q} t} \\
P_{\mathrm{i}}^{ \pm}=\mp \frac{i f_{1}^{\mathrm{e}}}{4 \pi}\left(\mathbf{E}_{q}^{ \pm} \times \mathbf{M}\right)_{\mathrm{i}}+\frac{1}{4 \pi} \sum_{\mathrm{j} l m} f_{2 \mathrm{ij}}^{l m} M_{l} M_{m} E_{\mathrm{j}}^{ \pm} .
\end{gathered}
$$

Le dipôle électrique induit par l'interaction du premier ordre est linéaire en composante de l'aimantation.
L'expression vectorielle de ce terme représente les propriétés gyroélectriques du cristal. Les interactions M. O. du second ordre sont décrites par un tenseur de quatrième rang $f_{\mathrm{ij}}^{l m}$ ayant 81 composantes.

Dans le cas d'un cristal cubique, on montre en prenant les axes du cube pour référentiel, que les effets du second ordre sont décrits par trois constantes M. O. indépendantes définies en notations de Voigt $\operatorname{par} f_{11} f_{12}$ et $f_{44}$ tel que :

$$
\left|\begin{array}{l}
\varepsilon_{11}^{\prime} \\
\varepsilon_{22}^{\prime} \\
\varepsilon_{33}^{\prime} \\
\varepsilon_{12}^{\prime} \\
\varepsilon_{13}^{\prime} \\
\varepsilon_{23}^{\prime} \\
\varepsilon_{21}^{\prime} \\
\varepsilon_{31}^{\prime} \\
\varepsilon_{32}^{\prime}
\end{array}\right|=\left|\begin{array}{cccccc|ccc}
f_{11} & f_{12} & f_{12} & 0 & 0 & 0 & 0 & 0 & 0 \\
f_{12} & f_{11} & f_{12} & 0 & 0 & 0 & 0 & 0 & 0 \\
f_{12} & f_{12} & f_{11} & 0 & 0 & 0 & 0 & 0 & 0 \\
0 & 0 & 0 & f_{44} & 0 & 0 & f_{44} & 0 & 0 \\
0 & 0 & 0 & 0 & f_{44} & 0 & 0 & f_{44} & 0 \\
0 & 0 & 0 & 0 & 0 & f_{44} & 0 & 0 & f_{44} \\
0 & 0 & 0 & f_{44} & 0 & 0 & f_{44} & 0 & 0 \\
0 & 0 & 0 & 0 & f_{44} & 0 & 0 & f_{44} & 0 \\
0 & 0 & 0 & 0 & 0 & f_{44} & 0 & 0 & f_{44}
\end{array}\right| \quad\left|\begin{array}{l}
M_{1} M_{1} \\
M_{2} M_{2} \\
M_{3} M_{3} \\
M_{1} M_{2} \\
M_{1} M_{3} \\
M_{2} M_{3} \\
M_{2} M_{1} \\
M_{3} M_{1} \\
M_{3} M_{2}
\end{array}\right|
$$

On en déduit aisément une expression vectorielle du dipôle électrique tel que :

$$
\begin{aligned}
P_{\mathrm{i}}^{ \pm}(2)=\frac{1}{4 \pi}\left[f_{12} \mathbf{M}^{2} E_{\mathrm{i}}^{ \pm}+2 f_{44}\right. & \left(\mathbf{M} \cdot \mathbf{E}^{ \pm}\right) \times \\
& \left.\times M_{\mathrm{i}}+\Delta f M_{\mathrm{i}}^{2} E_{\mathrm{i}}^{ \pm}\right] .
\end{aligned}
$$

Le premier terme associé à $f_{12}$ est indépendant de la direction de l'aimantation par rapport au champ électrique du rayonnement. Cela n'est pas le cas pour le second, terme associé à $f_{44}$, qui est maximal quand $\mathbf{M}$ est parallèle à $\mathbf{E}$. Le troisième terme représente l'anisotropie des effets M. O. du second ordre avec

$$
\Delta f=f_{11}-f_{12}-2 f_{44} \text {. }
$$

Quand $\Delta f=0$ les interactions du second ordre sont isotropes et décrites seulement par deux constantes M. O. $f_{12}$ et $f_{44}$. Le cristal dans ce cas est uniaxe avec l'axe optique parallèle à la direction de l'aimantation. Si $\Delta f \neq 0$, les effets du second ordre sont anisotropes et décrits par trois constantes $f_{11}, f_{12}$ et $f_{44}$. Dans ce cas 
le cristal devient biaxe. On définit un coefficient d'anisotropie $\rho$ des effets du second ordre par :

$$
\rho=2 f_{44} /\left(f_{11}-f_{12}\right) \text {. }
$$

A partir de l'équation d'évolution de l'aimantation totale $\mathbf{M}=\mathbf{M}+\mathbf{m}_{k}+\mathbf{m}_{q}$ du cristal soumise au champ magnétique total $\mathbf{H}=\mathbf{H}_{0}-\alpha \mathbf{M}+h_{q}$ où $M_{0}$, $m_{k}$ et $\alpha$ sont les aimantations statique et dynamique d'onde de spin $\omega_{k}$ et le coefficient de champ moléculaire dû à l'interaction d'échange, on montre que le rayonnement $\mathbf{h}_{q}$ induit dans la bande optique un dipôle (de caractère dispersif) $\mathbf{m}_{q}$ de composantes $m_{q}^{ \pm}$:

$$
\mathbf{m}_{q, k}^{ \pm}= \pm \frac{i f_{1}^{\mathbf{m}}}{4 \pi \varepsilon_{\mathrm{r}}}\left(\mathbf{h}_{q}^{ \pm} \times \mathbf{M}_{k}\right)
$$

avec

$$
M_{k}=M_{0}+m_{k} ; \quad \omega_{q k}=\omega_{q} \quad \text { ou } \quad \omega_{q} \pm \omega_{k}
$$

et

$$
f_{1}^{\mathrm{m}}=4 \pi|\gamma| \varepsilon_{\mathrm{r}} \omega_{q, k}^{-1}
$$

De ce dipôle on déduit facilement la partie hermitique du tenseur de susceptibilité magnétique qui montre l'existence d'un gyromagnétisme (représenté par le produit vectoriel) important dans la bande visible. A cause de la grande différence entre les énergies des photons visibles et IR et la séparation énergétique des sous-niveaux de base des ions magnétiques, les transitions réelles qui produisent l'absorption ne peuvent apparaître. Ainsi seule la partie anti-hermitique du tenseur de susceptibilité magnétique est nulle dans ce cas.

3.3 Propagation de la lumière dans UN MilieU MAGNÉTIQUE : BIRÉFRINGENCES MAGNÉTIQUES CIRCULAIRE ET LINÉAIRE. - L'introduction dans les équations de Maxwell des dipôles électriques et magnétiques limités aux termes linéaires:

$$
\begin{aligned}
& \mathbf{q} \times \mathbf{E}^{-}=\frac{\omega_{q}}{c}\left[\mathbf{h}^{-}-i \varepsilon_{\mathrm{r}}^{-1} f_{1}^{\mathrm{m}}\left(\mathbf{h}^{-} \times \mathbf{M}\right)\right] \\
& \mathbf{q} \times \mathbf{h}=-\frac{\omega_{q}}{c}\left[\varepsilon_{\mathrm{r}} \mathbf{E}^{-}+i f_{1}^{\mathrm{e}}\left(\mathbf{E}^{-} \times \mathbf{M}\right)\right]
\end{aligned}
$$

fait, après solution de ce système où on suppose une propagation suivant la direction de l'aimantation statique $M_{z}$, apparaître deux modes propres $q_{\alpha}$ et $q_{\beta}$ :

$$
\begin{aligned}
& \underset{\substack{\alpha \\
q_{\alpha}}}{2} q_{0}^{2}\left[\varepsilon_{\mathrm{r}} \mp\left(f_{1}^{\mathrm{m}}-f_{1}^{\mathrm{e}}\right) M_{0}-\varepsilon_{\mathrm{r}}^{-1} f_{1}^{\mathrm{e}} f_{1}^{\mathrm{m}} M_{0}^{2}\right]=q_{\mathrm{g}}^{2} \\
& \text { où } q_{0}=\omega_{q} / c .
\end{aligned}
$$

On vérifie que les modes propres correspondant à $q_{\alpha}$ et $q_{\beta}$ sont respectivement des ondes polarisées circulaires droites (PCD) $\left(E_{x}^{-}=-i E_{y}^{-}\right)$et circulaire gauches $(\mathrm{PCG})\left(E_{x}^{-}=+i E_{y}^{-}\right)$. Ces modes propres sont à l'origine de la biréfringence magnétique circulaire qui est responsable du pouvoir rotatoire magnétique ou rotation Faraday.

Les deux composantes PCD et PCG équivalentes à un rayonnement incident polarisé linéairement subissent l'une par rapport à l'autre un déphasage progressif tel qu'après un chemin de parcours $l$ on ait :

$$
\varphi_{\mathrm{g}}(l)-\varphi_{\mathrm{d}}(l)=\left(q_{\mathrm{g}}-q_{\mathrm{d}}\right) l .
$$

La polarisation linéaire résultante subit donc une rotation spatiale par unité de longueur de parcours appelée rotation Faraday spécifique $\varphi_{F}$ tel que :

$$
\varphi_{\mathrm{F}}=\frac{1}{2}\left(q_{\mathrm{g}}-q_{\mathrm{d}}\right) .
$$

En explicitant cette relation à partir de l'expression de $q_{\alpha}$ et $q_{\beta}$ où le terme quadratique

$$
\left(f_{1}^{\mathrm{e}} f_{1}^{\mathrm{m}} M^{2} \simeq 10^{-6} \times 10^{4}=10^{-9}\right)
$$

est négligeable devant les termes linéaires

et

$$
\left(-f_{1}^{\mathrm{e}} M \simeq 10^{-6} \times 10^{2}=10^{-4}\right.
$$

$$
\left.f_{1}^{\mathrm{m}} M \simeq 10^{-7} \times 10^{2}=10^{-5}\right),
$$

on obtient :

$$
\varphi_{\mathrm{F}}=\varphi_{\mathrm{e}}+\varphi_{\mathrm{m}}=-\frac{\omega_{q}}{2 \bar{n} c}\left(f_{1}^{\mathrm{e}}-f_{1}^{\mathrm{m}}\right) M_{z} .
$$

Les constantes $f_{1}^{\mathrm{e}}$ et $f_{1}^{\mathrm{m}}$ montrent que le cristal possède des propriétés gyroélectriques et gyromagnétiques. Dans la bande optique les matériaux sont donc bigyrotropes. La rotation « électrique » $\varphi_{\mathrm{e}}$ dépend étroitement des transitions dipolaires électriques (et donc de la fréquence) dans la bande visible, aussi ce terme est-il appelé rotation "dispersive ». La rotation magnétique $\varphi_{\mathrm{m}}$, étant associée aux transitions dipolaires magnétiques apparaissant dans la bande des microondes, possède une dispersion constante dans la bande visible. Pour cette raison $\varphi_{\mathrm{m}}$, qui est toujours positive (avec les conventions de polarisation adoptées ici), est parfois appelée rotation "non dispersive ", comme indiqué par ailleurs par l'expression suivante :

$$
\varphi_{\mathrm{m}}=2 \pi \bar{n} c^{-1}|\gamma| M_{z}
$$

où $f_{1}^{\mathrm{m}}$ a été explicité. Dans un cristal ferrimagnétique à deux sous-réseaux, on peut écrire :

$$
\varphi_{\mathrm{m}}=2 \pi \bar{n} c^{-1}\left(\gamma_{1} M_{1}-\gamma_{2} M_{2}\right)
$$

La propagation du rayonnement électromagnétique dans un cristal ayant des propriétés $M$. O. des premier et second ordres est décrite en introduisant dans les équations de Maxwell précédentes le dipôle $\mathbf{P}$ induit au second ordre M. O. Pour des directions du vecteur d'onde $\mathbf{q}$ et de l'aimantation statique $M_{\mathrm{s}}$ arbitraires par rapport aux axes cristallographiques, il devient très difficile de déterminer les modes propres de propagation.

Pour simplifier la discussion nous supposons que $\mathbf{q} / / \mathbf{M}_{z}$ et que $\Delta f=0$ (isotropie des effets $\mathbf{M}$. O. du second ordre). Dans ce cas les équations de Maxwell, dans lesquelles on a introduit le dipôle $\mathbf{P}(2)$ 
défini plus haut, font apparaître les deux modes propres $q_{\alpha}$ et $q_{\beta}$ suivants :

$$
\begin{aligned}
& q_{\alpha}^{2} \simeq q_{0}^{2}\left[\varepsilon_{\mathrm{r}}+f_{12} M_{z}^{2}\right]=q_{\perp}^{2} \\
& q_{\beta}^{2} \simeq q_{0}^{2}\left[\varepsilon_{\mathrm{r}}+\left(f_{12}+2 f_{44}\right) M_{z}^{2}\right]=q_{\|}^{2}
\end{aligned}
$$

qui correspondent à des modes de propagation polarisés linéairement suivant des directions ou parallèle $\left(q_{\alpha}\right)$ ou perpendiculaire $\left(q_{\beta}\right)$ à $M_{z}$. Au cours de la propagation dans un milieu $M$. O. quadratique la polarisation des modes $\alpha$ et $\beta$ reste linéaire, donc inchangée. Cette situation n'est plus valable dans le cas d'une polarisation linéaire incidente intermédiaire aux deux cas précédents. Ainsi pour une polarisation incidente à $\pi / 4$ de $\mathbf{M}_{z}$, l'apparition progressive d'un déphasage dans le temps entre les deux composantes parallèle $\mathbf{E}_{\|}$et perpendiculaire $\mathbf{E}_{\perp}$ à l'axe optique $O z$, produit une ellipticité $\bar{e}(\delta)$ dans la lumière transmise. Nous définirons la biréfringence magnétique linéaire par un déphasage spécifique Cotton-Mouton tel que :

$$
\varphi_{\mathrm{CM}}=q_{\|}-q_{\perp}=\frac{\omega_{q}}{c}\left(n_{\|}-n_{\perp}\right)=\frac{\omega_{q}}{\bar{n} c} f_{44} M_{z}^{2} .
$$

Avec les hypothèses simplificatrices adoptées $(\Delta f=0)$, on constate que la biréfringence Cotton-Mouton est décrite à partir de la constante $f_{44}$ uniquement. On montre que ce résultat se retrouve dans des milieux anisotropes $(\Delta f \neq 0)$ pour certaines géométries expérimentales particulières.

3.4 DESCRIPTION MACROSCOPIQUE QUANTIQUE DES EFFETS MAGNÉTO-OPTIQUES : DIFFUSIONS ÉLASTIQUES ET INÉLASTIQUES SPIN-PHOTONS. - De la densité d'énergie électromagnétique présente dans le cristal :

$$
W=\frac{1}{8 \pi}(\mathbf{E D}+\mathbf{h B}),
$$

on déduit dans une description classique les densités d'Hamiltonien d'interaction dipolaire électrique et magnétique tel que :

$$
\begin{aligned}
& H^{\mathrm{e}}=-\frac{1}{2} \sum_{q}\left(\mathbf{E}_{q}^{+} \mathbf{P}_{q}^{-}+\mathbf{E}_{q}^{-} \mathbf{P}_{q}^{+}\right) \\
& H^{\mathrm{m}}=-\frac{1}{2} \sum_{q}\left(\mathbf{h}_{q}^{+} \mathbf{m}_{q}^{-}+\mathbf{h}_{q}^{-} \mathbf{m}_{q}^{+}\right)
\end{aligned}
$$

où $E^{-}$et $P^{-}$d'une part et $E^{+}$et $\mathrm{P}^{+}$d'autre part représentent dans le plan complexe la moitié des vecteurs de Fresnel du champ électrique et du dipôle électrique et de leur complexe conjugué respectivement. Il en est de même pour l'interaction dipolaire magnétique. L'intérêt de présenter les expressions précédentes en fonction de ces quantités devient apparent lorsqu'on effectue le passage des Hamiltoniens classiques aux Hamiltoniens quantiques. En effet dans ce dernier cas $E^{+}$et $E^{-}$(ou $h^{+}$et $h^{-}$) s'expriment directement en fonction des opérateurs de photons $b_{q}$ et $b_{q}^{+}$tandis que $m^{-}$et $m^{+}$sont fonction des quantités $M^{+}$et $M^{-}$qui sont associées aux opérateurs de magnons $c_{k}$ et $c_{k}^{+}$, comme décrit dans le chapitre Il. En introduisant les opérateurs de photons et de magnons dans $H^{\mathrm{e}}$ et $H^{\mathrm{m}}$, on déduit les Hamiltoniens de diffusions élastiques et inélastiques induites par les interactions dipolaires électriques et magnétiques. Sans mentionner tous les termes d'interaction possibles nous donnons, à titre d'exemple, l'Hamiltonien d'interaction spin-photon induite au premier ordre par les couplages dipolaires électriques [3] :

$$
\mathcal{H}_{1}^{\mathrm{e}}=\mathcal{H}_{z}^{\mathrm{e}}(1)+\mathcal{H}_{x y}^{\mathrm{e}}(1)
$$

avec

$$
\begin{aligned}
& \mathcal{H}_{x y}^{\mathrm{e}}(1)=\mathcal{H}_{S}^{\mathrm{e}}(1)+\mathcal{H}_{\mathrm{AS}}^{\mathrm{e}}(1) \\
& \mathfrak{H}_{z}^{\mathbf{e}}(1)=\sum_{1,2}-i F_{1 z}^{z}\left(\mathbf{e}_{1 x} \mathbf{e}_{2 y}-\mathbf{e}_{1 y} \mathbf{e}_{2 x}\right) \cdot b_{1} b_{2}^{+} \Delta\left(\mathbf{q}_{1}-\mathbf{q}_{2}\right)+\text { h. c. } \\
& \mathcal{H}_{S}^{\mathrm{e}}(1)=\sum_{q_{1}, q_{2}, k} F_{1 x}^{x}\left[\left(\mathbf{e}_{1 \pi} \mathbf{e}_{2 r}^{*}-\mathbf{e}_{1 r} \mathbf{e}_{2 \pi}\right) b_{1} b_{2}^{+} a_{k}^{+} \Delta\left(\mathbf{q}_{1}-\mathbf{q}_{2}-\mathbf{k}\right)+\left(e_{1 r}^{*} e_{2 \pi}-e_{1 \pi} e_{2 r}\right) b_{1}^{+} b_{2} a_{k}^{+} \Delta\left(\mathbf{q}_{2}-\mathbf{q}_{1}-\mathbf{k}\right)\right] \\
& \mathcal{H}_{\mathrm{AS}}^{\mathrm{e}}(1)=\mathcal{J}_{S}^{\mathrm{e}}(1)\left(r \rightarrow l \text { et } a_{k}^{+} \rightarrow a_{k}\right)
\end{aligned}
$$

où $F_{1 z}^{z}$ et $F_{1 x}^{x}$ sont des coefficients qui dépendent entre autres de l'indice de réfraction, de $f_{1}$ et $M_{0}$.

L'opérateur $b_{1} b_{2}^{+}$montre que l'Hamiltonien $\mathcal{H}_{z}^{\mathrm{e}}(1)$ représente une diffusion spin-photon élastique qui n'est autre que l'effet Faraday décrit à partir d'une description phénoménologique quantique nouvelle. Dans de tels processus un photon incident polarisé linéairement suivant $o x$ est diffusé avec une polarisation à $\pi / 2$ suivant $o y$. On montre que le calcul de probabilité de ces transitions donne la valeur de la rotation Faraday spécifique du cristal étudié [3]. Les opérateurs $b_{1} b_{2}^{+} a_{k}^{+}$et $b_{1} b_{2}^{+} a_{k}$ montrent que les Hamiltoniens $\mathscr{H}_{S}$ et $\mathscr{H}_{\mathrm{AS}}$ représentent les diffusions spin-photon inélastiques ou Raman à 1 magnon des types Stokes et anti-Stokes respectivement. On peut définir une susceptibilité Raman à 1 magnon $\chi_{\mathbf{R}_{1}}$ à partir de la puissance lumineuse diffusée $P_{S}$ et de la probabilité de ces transitions $W_{\mathbf{R}}$ tel que :

$$
P_{S}=\frac{1}{2} \omega_{q_{1}} \chi_{\mathrm{R}_{1}} E_{q_{1}}^{2}=W_{\mathrm{R}_{1}} \hbar \omega_{q_{2}} V^{-1}
$$

Dans un milieu isotrope les coefficients Faraday $F_{1 z}^{z}$ et Raman $F_{1 x}^{x}$ sont définis à partir de la même constante magnéto-optique linéaire $f_{1}$. De cette remarque et en utilisant la règle d'or de Fermi, on 
montre sans difficulté que la susceptibilité Raman à 1 magnon est proportionnelle au carré de la rotation Faraday spécifique $\varphi_{F}$ tel que :

$$
\chi_{\mathrm{R}_{1}}=\left(\frac{c \lambda_{0}}{2 \pi^{2}}\right) \varphi_{\mathrm{F}}^{2} \sum_{k} \frac{n_{k} g \mu_{\mathrm{B}}}{\eta_{k} M_{0} V}
$$

où $n_{k}$ et $\eta_{k}$ sont la population et le taux de relaxation du magnon $\hbar \omega_{k}, \lambda_{0}$ est la longueur d'onde du rayonnement dans le vide. Cette susceptibilité est de l'ordre de $10^{-16}$ et $10^{-7}$ avec des magnons thermiques et des magnons cohérents excités par pompages radiofréquences «perpendiculaire » ou "parallèle » [3].

En explicitant le dipôle électrique $P(2)$ dans l'expression de $H^{\mathrm{e}}$, on obtient l'expression générale de l'Hamiltonien d'interaction magnéto-optique du second ordre induite par les transitions dipolaires électriques :

$\operatorname{He}^{\mathrm{e}}(2)=-\frac{1}{8 \pi} \int_{V} \sum_{\mathrm{ij} l m q_{1} q_{2}} f_{2 \mathrm{ij}}^{l m} M_{l} M_{m} E_{\mathrm{i}}^{-}\left(q_{1}\right) E_{\mathrm{j}}^{+}\left(q_{2}\right)+$ + herm. conj.

Sans entrer dans le détail des calculs, nous pouvons vérifier en introduisant les opérateurs de photon et de magnon que trois termes typiques associés à $M_{z}^{2}$; $M_{x} M_{y}$ (où $M_{x}^{2}$ et $M_{y}^{2}$ ) et $M_{z} M_{x}$ (ou $M_{z} M_{y}$ ) apparaissent

10 $M_{z}^{2} f_{2 z z, y y}^{z z}$

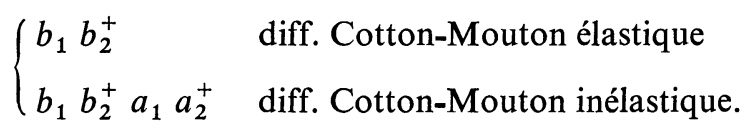

2o $M_{x} M_{y} f_{2 \mathrm{ij}}^{x y}$

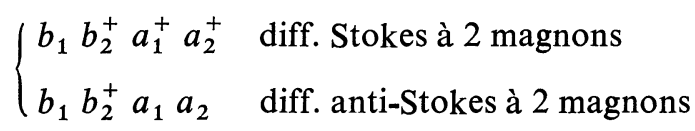

$$
\begin{aligned}
& M_{x}^{2} f_{2 \mathrm{ij}}^{x x} \\
& \left\{\begin{array}{llll}
b_{1} & b_{2}^{+} & a_{1}^{+} & a_{2}^{+} \\
b_{1} & b_{2}^{+} & a_{1} & a_{2} \\
b_{1} & b_{2}^{+} & a_{1} & a_{2}^{+}
\end{array} \quad \text { id. cas } 1 .\right.
\end{aligned}
$$

30 $M_{z} M_{x} f_{2 \mathrm{ij}}^{z x}$

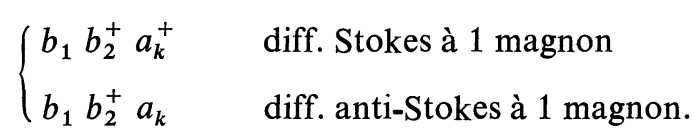

Les expressions exactes des Hamiltoniens associés aux différents cas décrits ci-dessus peuvent être déterminées sans difficultés. Dans un milieu isotrope, on montre que $\chi_{\mathbf{R}_{2}}$ est proportionnel au carré de la biréfringence magnétique linéaire spécifique ou « déphasage Cotton-Mouton spécifique $» \varphi_{\mathrm{CM}}$ comme défini précédemment.

Dès lors les susceptibilités Raman à 1 et 2 magnons peuvent être comparées et pour le processus Stokes par exemple on obtient :

$$
\left(\frac{\chi_{\mathrm{R}_{2}}}{\chi_{\mathrm{R}_{1}}}\right)_{\text {Stokes }}=\left(\frac{\varphi_{\mathrm{CM}}}{\varphi_{\mathrm{F}}}\right)^{2} \cdot \sum_{k} \frac{\left(n_{k}+1\right) g \mu_{\mathrm{B}}}{4 M_{0} V} .
$$

3.5 DESCRIPTION MICROSCOPIQUE DES EFFETS MAGNÉTO-OPTIQUES. - Une description microscopique simple des effets $M$. O. peut être donnée sur la base des transitions réelles et virtuelles discutée précédemment (section 2.3). Nous présentons successivement les diffusions élastiques et inélastiques spin-photon en considérant les transitions permises par les règles de sélection habituelles entre un multiplet de base ${ }^{2} \mathrm{~S}_{1 / 2}$ et un multiplet orbital excité ${ }^{2} \mathrm{P}_{3 / 2}$.

3.5.1 Diffusions élastiques $d u 1^{\mathrm{er}}$ ordre: effet Faraday. - Si on suppose seul le niveau inférieur de l'état $\mid{ }^{2} \mathrm{~S}_{1 / 2}>$ occupé, un rayonnement électromagnétique se propageant le long du champ magnétique (axe $o z$ ) induit uniquement les transitions $a$ et $f$ (transitions $\sigma$ ) du diagramme de Zeeman (Fig. 27).
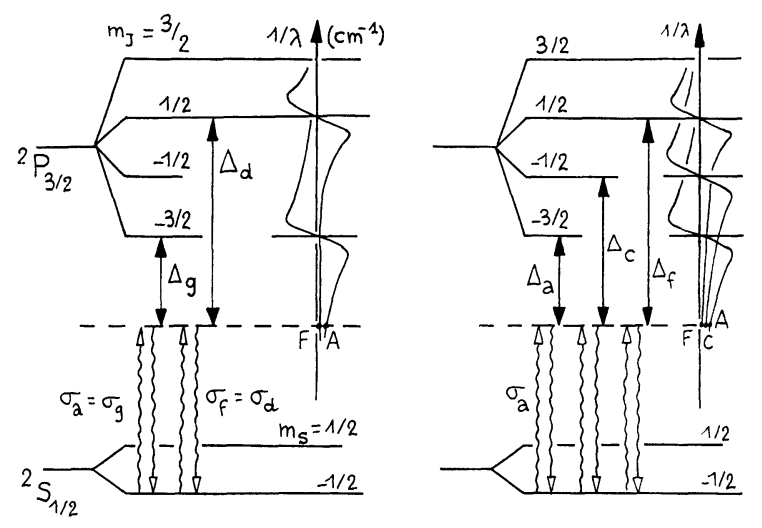

FIG. 27. - $-a$ ) Biréfringence magnétique circulaire. b) Biréfringence magnétique linéaire.

Dans le cas de transitions virtuelles, les séparations énergétiques associées aux composantes $a$ (ou $\sigma_{\mathrm{g}}$ ) et $f\left(\right.$ ou $\sigma_{\mathrm{d}}$ ) sont respectivement $\Delta_{\mathrm{g}}$ et $\Delta_{\mathrm{d}}$, soit donc une dispersion plus importante (point A) pour la composante $\sigma_{\mathrm{g}}$. Une différence d'indice apparaît pour ces composantes, ce qui entraîne la rotation du plan de polarisation de leur résultante : c'est la biréfringence circulaire ou rotation Faraday. La différence d'absorption des composantes $\sigma_{\mathrm{d}}$ et $\sigma_{\mathrm{g}}$ définit le dichroïsme magnétique circulaire.

3.5.2 Diffusion élastique du second ordre: effet Cotton-Mouton. - Si on suppose encore le niveau inférieur de l'état de base ${ }^{2} S_{1 / 2}$ uniquement occupé, un rayonnement électromagnétique se propageant perpendiculairement à l'axe $O z$ induit seulement les transitions $a$ et $f$ du diagramme de Zeeman si la polarisation des photons incidents est perpendiculaire à $O z$, et la transition $c$ si la polarisation est parallèle à $O z$. Les transitions virtuelles associées à ces composantes possèdent des dispersions différentes (points $\mathrm{A}$, 
$\mathrm{C}$ et $\mathrm{F}$ de la figure 27) qui produisent des indices $n_{\|}$ et $n_{\perp}$ différents. C'est l'origine de la biréfringence magnétique linéaire ou effet Cotton-Mouton.

Si les différents niveaux du multiplet de base sont peuplés, il faut, pour les effets Faraday et CottonMouton, tenir compte de toutes les transitions permises par les règles de sélection.

3.5.3 Diffusion spin-photon inélastique : effets Raman de spin et de magnon. - En couplant l'état de base avec un état excité, une transition virtuelle peut dans un ion modifier l'énergie finale de son état de base. Cette modification apparaît par le changement du nombre quantique magnétique $M_{S}$ de l'état de base après réémission du photon comme indiqué sur la figure 28. Ces transitions virtuelles peuvent augmenter

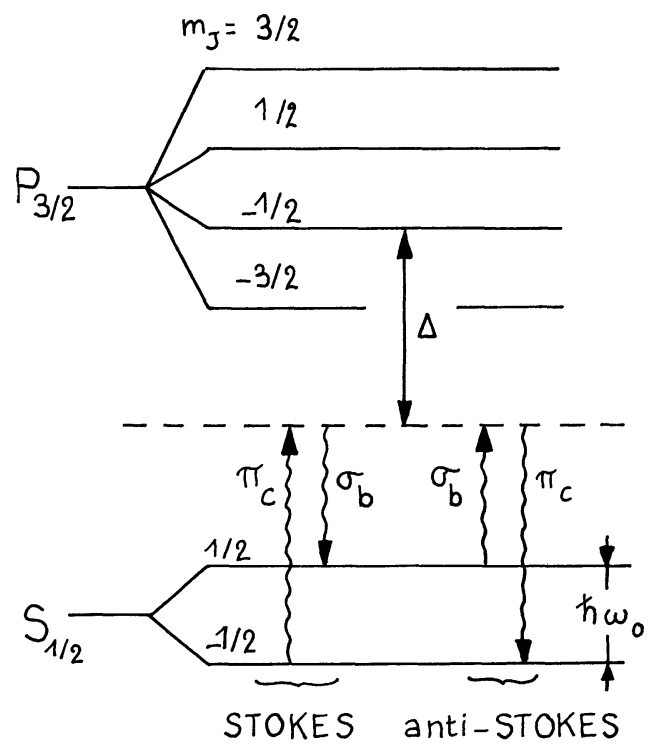

FIG. 28. - Effet Raman de Spin.

(diffusion Stokes) ou diminuer (diffusion anti-Stokes) l'énergie du niveau de base d'une quantité $\hbar \omega_{0}$. Une telle variation $\Delta M_{\mathrm{s}}= \pm 1$ dans l'état de base est possible grâce au couplage spin-orbite $\lambda^{\prime} \mathbf{L S}$ dans l'état excité qui mélange les mouvements orbitaux et de spin. En effet, les fonctions propres d'un état excité $\mid J, M_{J}>$ en présence de l'interaction spinorbite peuvent se définir à partir d'une combinaison linéaire des fonctions propres non perturbées $\mid M_{L} M_{S}>$ :

$$
\begin{aligned}
\left|L S J M_{J}>=\sum_{M_{L} M_{S}}\right| L S M_{L} M_{S}> & \times \\
& \times<L S M_{L} M_{S} \mid L S J M_{J}>.
\end{aligned}
$$

Donc au cours d'une transition virtuelle la capture d'un photon connecte dans un premier temps l'état de base, de nombre quantique magnétique $M_{S}$ à l'un des états excités $\mid M_{L}, M_{S}>$ contenu dans $\mid J, M_{J}>$. Dans un deuxième temps l'opérateur $\lambda^{\prime} L^{ \pm} S^{\mp}$ de l'interaction spin-orbite mélange (ou couple) ces états excités tel que :

$$
L^{ \pm} S^{\mp} \mid M_{L} M_{S}>=\text { Cte } \mid M_{L} \pm 1, M_{S} \mp 1>\text {. }
$$

On observe dès lors une transition virtuelle inélastique lorsque le photon réémis correspond à une transition entre l'état excité $\mid M_{L} \pm 1, M_{S} \pm 1>$ et le niveau de base $\mid 0, M_{S} \pm 1>$; c'est l'effet Raman de spin du premier ordre. De la même manière une transition virtuelle peut produire dans le multiplet de base une variation $\Delta M_{S}= \pm 2$ qui correspond à une diffusion Raman de spin du second ordre. Dans un milieu ferro-, ferri- ou antiferromagnétique, à cause du couplage d'échange, l'excitation locale d'un spin unique est impossible. Dès lors, bien que la transition virtuelle reste localisée sur un seul ion du cristal, l'excitation magnétique finale $\Delta M_{S}= \pm 1, \pm 2$ est étendue, par le champ d'échange, à l'ensemble des $N$ spins ioniques de cristal qui subissent chacun une variation infime $\Delta M_{S} / N$. Dans ce cas la diffusion spin-photon inélastique crée ou absorbe un ou plusieurs quanta d'onde de spin ou magnons : c'est la diffusion Raman à 1 ou 2 magnons,

3.5.4 Diffusions élastiques et inélastiques «magnétiques ». - Tous les processus décrits ci-dessus impliquent une transition induite par le couplage dipolaire électrique entre deux états orbitaux différents. En fait le couplage dipolaire magnétique produit des effets identiques, à partir de transitions entre les différents états magnétiques du multiplet de base. La contribution de ces couplages magnétiques aux effets MO est négligée par la plupart des auteurs, dans la bande optique, ce qui est loin d'être justifié dans certains matériaux. Il est possible de discuter quantitativement les origines microscopiques des interactions spinphoton à partir du calcul quantique de la polarisabilité dynamique tensorielle d'un ion ou d'un ensemble d'ions magnétiques tel que la susceptibilité diélectrique $\chi_{\mathrm{ij}}^{+}$s'écrive :

$$
\chi_{\mathrm{ij}}^{+}=\sum_{a} N_{a} \alpha_{\mathrm{ij}}^{+}(a)
$$

où $\alpha_{\mathrm{ij}}(a)$ est un élément du tenseur de polarisabilité dynamique d'un ion « $a$ ». L'Hamiltonien total qui décrit le cristal magnétique est défini par $H$ tel que :

$$
H=H_{1}+H_{\mathrm{R}}
$$

avec

$$
\begin{aligned}
H_{1} & =H_{0}+H_{\mathrm{cr}}+H_{\text {éch }}+H_{\mathrm{SO}}+H_{\mathrm{Z}} \\
H_{\mathrm{R}} & =-\mathbf{E P}=-\mathrm{erE} \\
& =-\mathrm{e}\left(\mathbf{r}^{+}+\mathbf{r}^{-}\right)\left(\mathbf{E}^{+} \mathrm{e}^{-i \omega_{q} t}+\mathbf{E}^{-} \mathrm{e}^{i \omega_{q} t}\right)
\end{aligned}
$$

où les différents termes de $H_{1}$ représentent l'évolution $\mathrm{du}$ système électronique dans l'approximation du champ central $\left(H_{0}\right)$, le champ cristallin $\left(H_{\mathrm{cr}}\right)$, le champ d'échange $\left(H_{\mathrm{e}}\right)$, le couplage spin-orbite $\left(H_{\mathrm{so}}\right)$ et le champ magnétique appliqué ou terme de Zeeman 
$\left(H_{z}\right)$. En absence de rayonnement les états propres $\mid \mathrm{n}>$ du système sont donnés par :

$$
H_{1} \mid \mathrm{n}>=\hbar \omega_{\mathrm{n}} \mathrm{n}>\text {. }
$$

Par la perturbation $H_{\mathrm{R}}$, où on ne considère que l'interaction dipolaire électrique, un rayonnement électromagnétique induit un couplage (transitions réelles ou virtuelles) entre les états $|n\rangle$ tel que le système est maintenant décrit par les fonctions $\left|\psi_{\mathrm{n}}\right\rangle$ définies par:

$$
H\left|\psi_{\mathrm{n}}>=E_{\mathrm{n}}\right| \psi_{\mathrm{n}}>.
$$

Les processus d'absorption à un photon sont décrits par la théorie des perturbations linéaires en $H_{\mathrm{R}}$. Par contre la biréfringence magnétique circulaire, ou la diffusion Raman sont décrites par la théorie des perturbations quadratique en $H_{\mathrm{R}}$ et linéaire en $H_{\text {so }}$. Ces diffusions doivent ainsi, impliquer des perturbations du troisième ordre. La réponse $d u$ système magnétique à une excitation électromagnétique est donnée par la polarisabilité dynamique $\alpha(a)$ des ions magnétiques. On déduit la polarisabilité dynamique d'un ion $" a$ » du dipôle induit $\mathbf{P}$ dans l'état perturbé $\left|\psi_{\mathrm{g}}\right\rangle$ par :

$$
\begin{aligned}
<\mathbf{P}> & =\sum_{\mathbf{g}}<\psi_{\mathrm{g}}|\mathbf{P}| \psi_{\mathrm{g}}>\rho_{\mathrm{g}} \\
& =\sum_{\mathbf{g}}<\psi_{\mathrm{g}} \mid \text { er } \mid \psi_{\mathrm{g}}>\rho_{\mathrm{g}}
\end{aligned}
$$

où la sommation s'étend à tous les sous-niveaux du multiplet de base de probabilité d'occupation $\rho_{\mathrm{g}}$. Les composantes cartésiennes du dipôle induit s'écrivent encore :

$$
<P_{\mathrm{i}}^{ \pm}>=\sum_{\mathrm{j}} \alpha_{\mathrm{ij}}^{ \pm} E_{\mathrm{j}}^{ \pm} \mathrm{e}^{\mp i \omega_{q} t}
$$

A partir de la théorie des perturbations dépendant du temps, on obtient sans difficulté l'état de base perturbé par le rayonnement électromagnétique :

$$
\begin{array}{r}
\left|\psi_{\mathrm{g}}\right\rangle=\mid \mathrm{g}>\mathrm{e}^{-i \omega \mathrm{g} t}+\frac{e}{\hbar} \sum_{\mathrm{n}}\left[\left\langle\mathrm{n}\left|\mathbf{r E}^{+}\right| \mathrm{g}\right\rangle\left(\frac{\mathrm{e}^{i\left(\omega_{\mathrm{ng}}-\omega_{\mathrm{q}}\right) t}-1}{\omega_{\mathrm{ng}}-\omega_{q}}\right)+\right. \\
\left.+<\mathrm{n}\left|\mathbf{r E}^{-}\right| \mathrm{g}>\left(\frac{\mathrm{e}^{i\left(\omega_{\mathrm{ng}}+\omega_{\mathrm{q}}\right) t}-1}{\omega_{\mathrm{ng}}+\omega_{q}}\right)\right] \mid \mathrm{n}>\mathrm{e}^{-i \omega_{\mathrm{n}} t}
\end{array}
$$

où on a supposé qu'à l'instant initial seul l'état $|\mathrm{g}\rangle$ est occupé. A partir des trois dernières équations on montre que la polarisation dynamique associée à $E_{\mathbf{j}}^{+}$s'écrit :

$$
\begin{gathered}
\alpha_{\mathrm{ij}}^{+}=\frac{e^{2}}{\hbar} \sum_{\mathrm{g}, \mathrm{n}} \rho_{\mathrm{g}}\left\{\operatorname{Re}<\mathrm{g}\left|\mathbf{r}_{\mathrm{i}}\right| \mathrm{n}><\mathrm{n}\left|\mathbf{r}_{\mathrm{j}}\right| \mathrm{g}>\left[\zeta^{*}\left(\omega_{\mathrm{ng}}-\omega_{q}\right)+\zeta\left(\omega_{\mathrm{ng}}+\omega_{q}\right)\right]+\right. \\
\left.\quad+\operatorname{Im}<\mathrm{g}\left|\mathbf{r}_{\mathrm{i}}\right| \mathrm{n}><\mathrm{n}\left|\mathbf{r}_{\mathrm{j}}\right| \mathrm{g}>\left[\zeta^{*}\left(\omega_{\mathrm{ng}}-\omega_{q}\right)-\zeta\left(\omega_{\mathrm{ng}}+\omega_{q}\right)\right]\right\} \\
\left.\quad \zeta^{*}\left(\omega_{\mathrm{ng}}-\omega_{q}\right) \pm \zeta\left(\omega_{\mathrm{ng}}+\omega_{q}\right)\right]=\left[\frac{P}{\omega_{\mathrm{ng}}-\omega_{q}} \pm \frac{P}{\omega_{\mathrm{ng}} \omega_{q}}+i \pi \delta\left(\omega_{\mathrm{ng}}-\omega_{q}\right) \mp i \pi \delta\left(\omega_{\mathrm{ng}}+\omega_{q}\right)\right] .
\end{gathered}
$$

En fait, l'interaction rayonnement-atome est décrite, en toute rigueur, en remplaçant l'opérateur dipolaire électrique $r_{\mathrm{i}}$ par $R_{\mathrm{i}}$ tel que :

$$
R_{\mathbf{i}}=\left[\mathbf{r}+i(\mathbf{q} \cdot \mathbf{r}) \cdot \mathbf{r}-\frac{\hbar}{2 m \omega_{q}}(\mathbf{L}+2 \mathbf{S}) \times \mathbf{q}+\cdots\right]_{\mathrm{i}}
$$

où les second et troisième termes représentent les interactions quadrupolaire électrique et dipolaire magnétique respectivement. Ces équations constituent le point de départ de toute analyse microscopique des interactions spin-photon.

$\mathrm{Si}$ on suppose que $\mid \mathrm{g}>$ est l'état de base, seul occupé au temps initial, la polarisabilité $\alpha_{\mathrm{ij}}^{+}$associée à $E^{+}$comprend, par le facteur entre crochets, 4 termes représentant les composantes réelles et virtuelles de deux transitions décrites sur la figure 29 par les éléments de matrice : $\left\langle\mathrm{n}\left|E^{+} r^{-}\right| \mathrm{g}\right\rangle$ (transition 1) et $\left\langle\mathrm{g}\left|E^{+} r^{+}\right| \mathrm{n}\right\rangle$ (transition 2). La transition 2 étant toujours virtuelle, le quatrième terme $\delta\left(\omega_{\mathrm{ng}}+\omega_{q}\right)$ est toujours nul, le troisième terme $\delta\left(\omega_{\mathrm{ng}}-\omega_{q}\right)$ est non nul lorsque la transition 1 est résonnante ou réelle. Les premier et second termes décrivent la dispersion des transitions 1 et 2 respectivement. Ces termes sont à l'origine de la réfraction, des biréfringences naturelle et magnétique circulaire et linéaire et des diffusions Raman de spin et de magnon. En dehors des raies d'absorption seuls les termes dispersifs sont non négligeables et se limitent aux expressions suivantes :

$$
\begin{aligned}
& \frac{P}{\omega_{\mathrm{ng}}-\omega_{q}}+\frac{P}{\omega_{\mathrm{ng}}+\omega_{q}} \simeq \frac{1}{\omega_{\mathrm{ng}}-\omega_{q}}+\frac{1}{\omega_{\mathrm{ng}}+\omega_{q}}= \\
& =\frac{2 \omega_{\mathrm{ng}}}{\omega_{\mathrm{ng}}^{2}-\omega_{q}^{2}} \\
& \frac{P}{\omega_{\mathrm{ng}}-\omega_{q}}-\frac{P}{\omega_{\mathrm{ng}}+\omega_{q}} \simeq \frac{2}{\omega_{\mathrm{ng}}^{2}-\omega_{q}^{2}} .
\end{aligned}
$$

Ce sont ces expressions qui, par le terme $\omega_{\text {ng }}$, introduisent toutes les perturbations magnétiques (interactions spin-orbite d'échange et de Zeeman) de non magnétiques (champ cristallin) responsables des diffusions élastiques et inélastiques spin-photon et des 

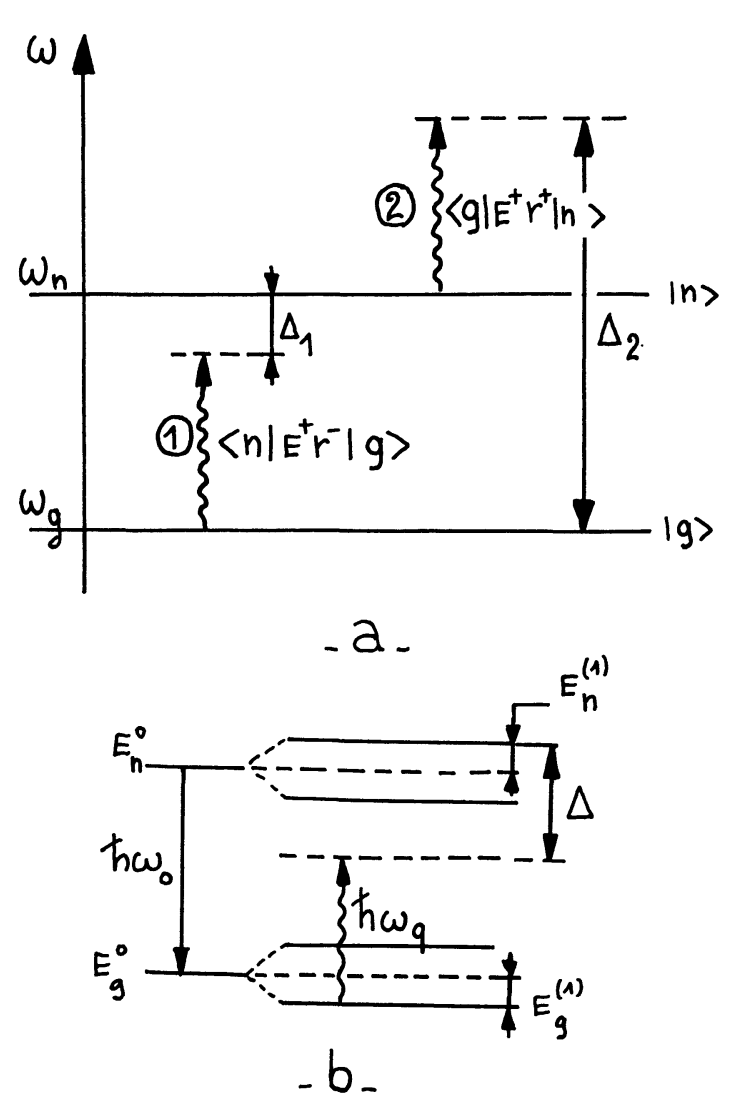

FIG. 29. diffusions non magnétiques. En effet, faisons l'hypothèse simplificatrice suivante pour les niveaux d'énergie des ions magnétiques (Fig. 29b) :

$$
\left|E_{\mathrm{g}}^{(1)}\right|,\left|E_{\mathrm{n}}^{(1)}\right| \ll \hbar \omega_{q} \ll \hbar \omega_{0}=E_{\mathrm{n}}^{0}-E_{\mathrm{g}}^{0}
$$

où $E_{\mathrm{g}}^{(1)}$ et $E_{\mathrm{n}}^{(1)}$ sont les corrections énergétiques aux niveaux non perturbés $E_{\mathrm{g}}^{0}$ et $E_{\mathrm{n}}^{0}$ produites par les perturbations des champs cristallin, d'échange, du couplage spin-orbite et du champ magnétique appliqué.

Les facteurs de fréquence 9 issus de la fonction zéta de Dirac peuvent s'exprimer sous forme de développements limités tel qu'on ait par exemple :

$$
\begin{aligned}
\frac{P}{\omega_{\mathrm{ng}}-\omega_{q}} & =\frac{P}{\omega_{\mathrm{ng}}+\omega_{q}} \simeq \frac{2}{\omega_{\mathrm{ng}}^{2}-\omega_{q}^{2}} \\
& \simeq \frac{2}{\omega_{0}^{2}-\omega_{q}^{2}}\left[1-\frac{2 \omega_{0}\left(E_{\mathrm{n}}^{(1)}-E_{\mathrm{g}}^{(1)}\right)}{\hbar\left(\omega_{0}^{2}-\omega_{q}^{2}\right)}+\cdots\right] .
\end{aligned}
$$

L'expression entre crochets contient des termes en puissances croissantes de $E_{\mathrm{n}}^{(1)}$ et $E_{\mathrm{g}}^{(1)}$ qui dépendent directement des opérateurs de spin par les interactions d'échange et spin-orbite tel que :

$$
\begin{aligned}
E_{\mathrm{n}}^{(1)}=H_{\mathrm{z}}+H_{\mathrm{crist}}+\sum_{a} \lambda^{\prime} \mathbf{L}_{a} \mathbf{S}_{a}+ & \sum_{a>b} J_{a b}^{\prime} \mathbf{S}_{a} \cdot \mathbf{S}_{b}+ \\
& +\sum_{a>b} J_{a b}^{\prime \prime} \mathbf{S}_{a} \times \mathbf{S}_{b}
\end{aligned}
$$

\section{TABLEAU II}

Ordre des perturbations
Eléments de matrice décrivant les transitions

\section{Effets}

Absorption 1 photon
effet Zeeman $\sigma_{\mathrm{d}} \sigma_{\mathrm{g}} \pi$

Diffusion élastique

$\mathrm{E}\left({ }^{*}\right)$

E

Faraday

Raman 1 magnon

$I\left({ }^{* *}\right)$
Type de diffusion $\begin{array}{ll}\text { Ordre } & \frac{\Delta M_{\mathrm{s}}}{g \mu_{\mathrm{B}}} \\ \text { M. O. }\end{array}$

0

0

0 $<\mathrm{g}^{\prime}\left|r_{\mathrm{i}}^{+}\right| \mathrm{n}^{\prime}>\times$ $\left.\times<\mathrm{n}^{\prime}\left|H_{\text {spin }}\right| \mathrm{n}><\mathrm{n}\left|r_{\mathrm{j}}^{-}\right| \mathrm{g}\right\rangle$

$$
\begin{aligned}
H_{\mathrm{spin}}=\lambda^{\prime} \mathbf{L}_{a} & \mathbf{S}_{a}=\lambda^{\prime} \mid L_{a}^{z} S_{a}^{z}+ \\
& +\frac{1}{2}\left(L_{a}^{+} S_{a}^{-}+L_{a}^{-} S_{a}^{+}\right)
\end{aligned}
$$

3$$
H_{\text {spin }}=J^{\prime} S_{a} S_{b}=J^{\prime} S_{a}^{z} S_{b}^{z}+
$$$$
+\frac{1}{2}\left(S_{a}^{+} S_{b}^{-}+S_{a}^{-} S_{b}^{+}\right)
$$

Raman 2 magnons

Cotton-Mouton

$$
H_{\mathrm{spin}}=\left(\lambda^{\prime} \mathbf{L}_{a} \mathbf{S}_{a}\right)^{2} \rightarrow\left\{\begin{array}{l}
\left(L_{a}^{z} S_{a}^{z}\right)^{2} \\
\left(L_{a}^{+} S_{a}^{-}\right)^{2} \\
L_{a}^{z} S_{a}^{z} L_{a}^{+} S_{a}^{-}
\end{array}\right.
$$

Raman 2 magnons

Raman 1 magnon

$$
H_{\mathrm{spin}}=\left(J^{\prime} \mathbf{S}_{a} \mathbf{S}_{b}\right)^{2} \rightarrow\left(S_{a}^{+} S_{b}^{-}\right)^{2}
$$
Raman 4 magnons

1

(*) Elastique.

(**) Inélastique. 
où $\lambda^{\prime}, J^{\prime}$ et $J^{\prime \prime}$ sont les constantes spin-orbite, d'échange isotrope et d'échange anisotrope dans les états orbitaux excités. Nous concevons ainsi la possibilité d'obtenir une polarisabilité diélectrique dépendant du spin aux premier ordre, second ordre, etc... en explicitant l'expression générale de $\alpha_{i j}$ en fonction des relations précédentes.

La détermination des fonctions d'onde $|\mathrm{g}\rangle$ et $|\mathrm{n}\rangle$ des ions magnétiques est généralement délicate sinon impossible sauf dans le cas de certains ions paramagnétiques où un calcul de biréfringence ou dichroïsme magnétique circulaire pourrait s'envisager.

Pour résumer et conclure la discussion qui précède nous avons rassemblé dans le tableau suivant les éléments de matrice décrivant les différents effets M. O. en spécifiant les ordres de perturbation, les ordres M. O., la variation de l'état magnétique et le type des diffusions.

Cet article qui constitue une introduction aux interactions spin-photon pourra être utilement approfondi en consultant par exemple les ouvrages suivants :

Gyromagnétisme et résonance magnétique

Abragam, A. et Bleaney, B., Electron Paramagnetic Resonance (Edit. Marshall et Wilkin son).

\section{Ondes de spin et relaxation ferrimagnétique}

Sparks, M., Ferromagnetic Relaxation Theory (Mc Graw Hill Book Company).

PIRCHER, G., Ferrites et grenats phénomènes non linéaires (Dunod).

\section{Magnéto-optique}

LE GALL, H., Spin photon interactions in magnetic crystals. Magnetooptical effects : First Summer School on Magnetooptics, Podhradi (1973). (Edité par J. Kaczér, Academia Praha) 1974.

Le Gall, H., Jamet, J. P., Vien, T. K. et Desormière, B., Phys. Stat. Sol. (b) 46 (1971) 467 et Phys. Stat. Sol. (b) 47 (1971) 591.

Le Gall, H., J. Physique Collq. 32 (1971) C1-590.

\section{Bibliographie}

[1] Akhiezer, A. I., J. Phys. 10 (1946) 217.

[2] Kasuya, T. and Le Craw, R. C., Phys. Rev. Lett. 6 (1961) 223.

[3] Le Gall, H. et Jamet, J. P., Phys. Stat. Sol. (b) 46 (1971) 467. 